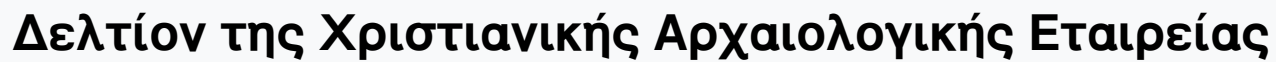

Tó 38 (2017)

$\Delta \varepsilon \lambda$ tíov XAE 38 (2017), Пعрíoঠos $\Delta^{\prime}$

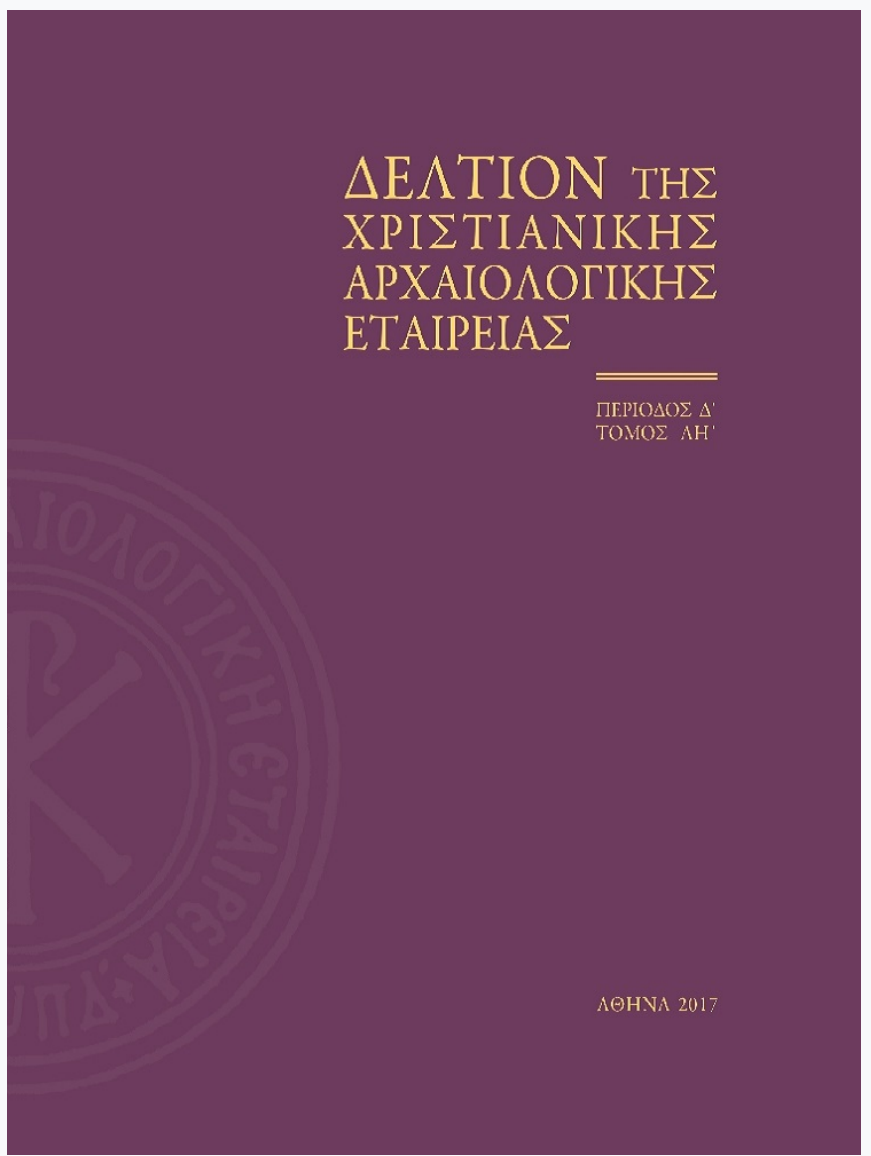

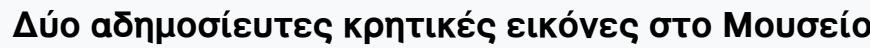

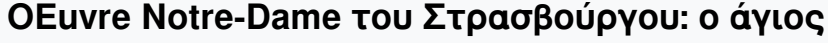

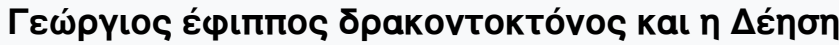

Nano CHATZIDAKIS (Navú XATZHAAKH)

doi: $10.12681 /$ dchae. 14225

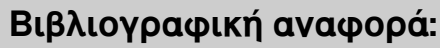

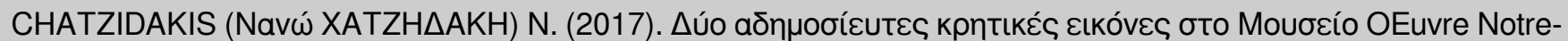

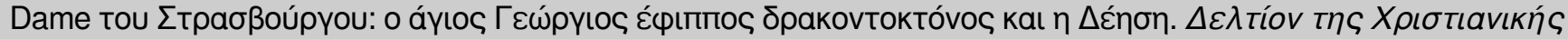

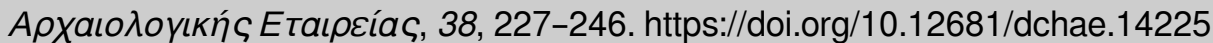




\section{Nano Chatzidakis}

\section{DEUX ICÔNES CRÉTOISES INÉDITES AU MUSÉE DE L'EUVRE NOTRE-DAME DE STRASBOURG : SAINT GEORGES A CHEVAL TERRASSANT LE DRAGON ET LA DÉISIS}

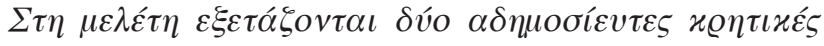

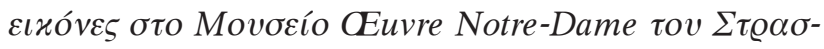
ßov́

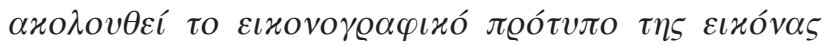

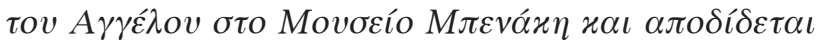

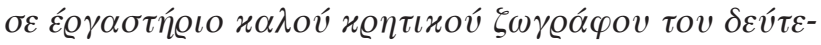

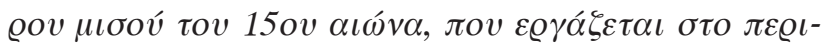

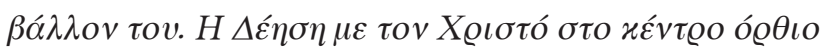

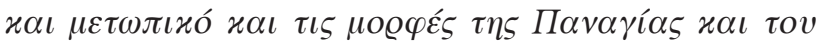

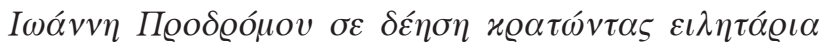

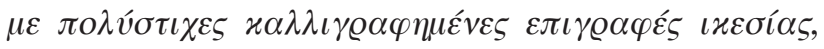

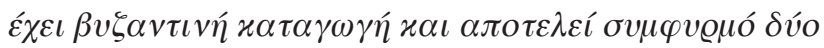

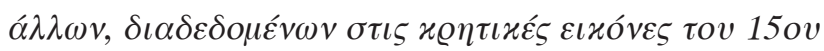

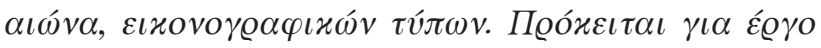

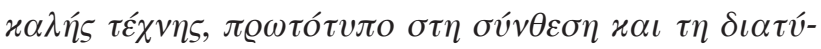

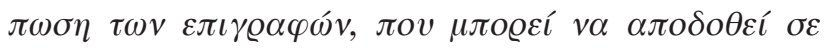

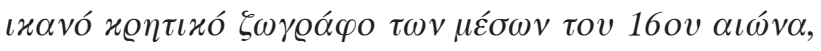

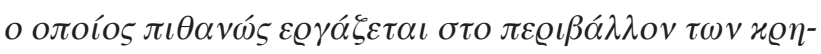

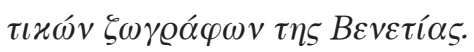

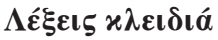

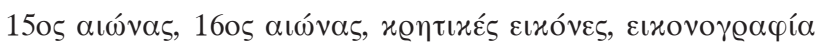

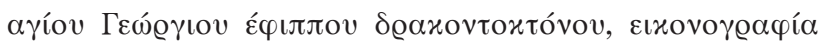

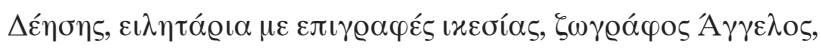

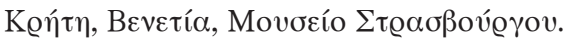

$\mathrm{P}$ armi les nombreuses icônes post-byzantines des collections publiques en France, seul un nombre restreint a attiré l'attention des spécialistes ${ }^{1}$. Dans cette étude ré-

\footnotetext{
* Professeur en retraite de l'Université d'Ioannina. nanochatzidakis $@ y a h o o . g r$

${ }^{1}$ Voir J. Durand (dir.), Byzance. L'art byzantin dans les collections
}

Two unpublished icons at the CEuvre Notre Dame Museum, Strasbourg are examined in the present study. Saint George the dragon-slayer on a horseback is painted after the iconographic model of the painter Angelos' icon at the Benaki Museum. It is attributed to an outstanding Cretan painter of the second half of the 15th century, working close to his workshop. The Deisis, where Christ at the center is represented, standing and frontal and the Virgin and Saint John the Forerunner turned in prayer, holding long unfolded scrolls with the text of the prayer of intercession, in calligraphic lettering, derives from Byzantine iconography and presents an original compilation of two other iconographic types of the Deisis, widely diffused on 15th century Cretan icons. It is a fine work, distinguished for its creative composition and the original rendering of the inscriptions; it can be attributed to a skillful Cretan painter, of the middle of the 16th century, working probably in the context of Cretan painters in Venice.

\section{Keywords}

15th century; 16th century; Cretan icons; iconography of St. George mounted and dragonslayer; iconography of the Deisis; inscribed scrolls with the prayer of intercession; painter Angelos; Crete; Venice; Strasbourg Museum.

digée en hommage à ma chère collègue et amie Catherine Jolivet, je vais présenter deux icônes crétoises inédites du Musée de l'EEuvre Notre-Dame de Strasbourg

publiques françaises (cat. exp.), Paris, musée du Louvre, 1992, nos 368, 371, 372, 373 et 374. N. Delsaux, "A propos de l'exposition Byzance, quelques exemples de restauration d'icônes”, La Revue du Louvre et des musées de France, 1992, no 5/6, p. 86-93, fig. 1-5, 
que j'ai eu la chance d'examiner lors de mon séjour dans cette ville en 1998 et de montrer sommairement dans une communication à Paris en $2001^{2}$ (Fig. 1 et 6).

\section{L'icône de saint Georges à cheval terrassant le dragon}

L'icône de saint Georges à cheval tuant le dragon fait partie du legs Gustave Schlumberger (1844-1929), effectué à Strasbourg le 23 février $1930^{3}$. Grand érudit, historien de Byzance, numismate et grand collectionneur de monnaies, de sceaux et d'objets d'art byzantin, Schlumberger avait fait plusieurs voyages en Orient latin, et entre autres en Grèce, à Athènes, à Patras et dans les îles (Rhodes) ${ }^{4}$; il avait encore visité l'Italie et en particulier

10, 13-15. A. Semoglou, "Saint Cyriaque et la Vraie Croix. Essai d'interprétation d'une icône crétoise du Louvre", La Revue du Louvre, 2001, no 5, p. 35-40, fig. 1. N. Chatzidakis, "Une icône crétoise de saint Georges à cheval au musée du Louvre. La diffusion $\mathrm{du}$ thème du saint cavalier "en parade "dans les icônes crétoises des XVe et XVIesiècles”, La Revue du Louvre 4 (2014), 58- 69.

${ }^{2}$ N. Chatzidakis, "Deux icônes crétoises inédites au musée de l'Euvre Notre-Dame de Strasbourg", XXe CIEB, Pré-Actes, III. Communications libres, Résumés, 19-25 Août 2001, Paris 2001, 362. Leur présence m'avait été signalée par ma collègue Marielle Martiniani-Reber, conservateur au Musée d'Art et d'Histoire de Genève, lorsque j'étais professeur invité à l'Université de Strasbourg, pendant le second semestre de 1998. Mme Cécile Dupeux, conservateur du Musée de l'Euvre Notre-Dame, avait eu la gentillesse de m'envoyer les photos et les diapositives utilisées dans la communication de 2001 et la présente publication.

${ }^{3}$ Legs Schlumberger : no 705, Rec. no R. 900 ; no. d'inventaire : 22.995.0.659.1. Anonyme, "Les donations testamentaires de G. Schlumberger, grand érudit et collectionneur", Journal des débats (21 janvier 1930), dans G. Schlumberger, Mes souvenirs 18841928 (éd. Adrien Blanchet), Paris 1934, 415. Il s'agit d'un legs dont l'importance avait été marquée par une exposition au Musée de la ville de Strasbourg: Des signes et des mots. L'écriture des signes dès origines au Moyen-âge : trésors inédits des collections strasbourgeoises (dirigée par F. Keller), 25.9.2003- 31.8.2004, Strasbourg 2003.

${ }^{4}$ G. Schlumberger, "Numismatique de Rhodes avant la conquête de l'île par les chevaliers de Saint-Jean”, Revue Archéologique XXX, nouv. série, 31 (janvier-juin 1876), 233-244. G. Schlumberger, "Fresques du XIVe siècle, d'un caveau funéraire de l'église de N.-D. de Philérémos (ou Philerme), à Rhodes”, MonPiot XIX (1911), 211-216.
Venise ${ }^{5}$. Il faut aussi retenir le fait que l'auteur de l'Epopée byzantine ${ }^{6}$, nommé docteur honoris causa de l'université d'Athènes, était lié d'amitié profonde avec Pénélope Delta (1874-1941), éminente personnalité des lettres grecques et sœur du fondateur du musée Bena$\mathrm{ki}^{7}$; il était également lié avec d'autres personnalités éminentes comme Adamantios Adamantiou ${ }^{8}$ et encore avec le chef de l'état hellénique Eleftherios Venizélos", qui avait assisté à la célébration pour la présentation des deux volumes dédicatoires qui lui avaient été remis à Paris, chez lui, le 30 novembre $1924^{10}$. En tenant compte des relations privilégiées de Gustave Schlumberger avec la Grèce, la présence de cette icône parmi les œuvres de ses collections n'est pas surprenante.

L'icône de saint Georges, de dimensions plutôt grandes (hauteur : $53,5 \mathrm{~cm}$; largeur : $46,5 \mathrm{~cm}$ ), se trouve en bonne condition sans avoir été restaurée dans le passé (Fig. 1). La surface peinte sur un fond doré porte les marques du temps sur le vernis et sur quelques petits dégâts aux confins de la partie haute et dans le bas. Une inscription en lettres rouges majuscules se distingue clairement, de part et d'autre de la tête du saint, avec le nom de saint Georges : [O] A(ГIOS) [ГЕ] RPГIOS.

Sur la surface dorée domine le jeune saint militaire, avec son manteau rouge flottant en arrière, monté sur

\footnotetext{
${ }^{5}$ Schlumberger, Mes souvenirs 1884-1928, op.cit., passim. "C'est souvent au cours de croisières en Méditerranée, sur son yacht, qu'il alliait visites archéologiques et de musées, acquisition d'objets rares destinés à enrichir ses collections" page éléctronique : INHA, Dictionnaire critique des historiens d'art, 9/2/2010 (Mireille Pastoureau). <http://www.inha.fr/fr/ressour ces/publications/publications -numeriques/dictionna ire-critique-des-historiens-de-l-art/schlumberger-leon-gustave.html>.

${ }^{6} \mathrm{G}$. Schlumberger, L'Epopée byzantine, à la fin du Xe siècle, I-III, Paris 1896-1905.

${ }^{7}$ Leur correspondance d'une durée de dix-sept ans (1910-1927), constitue un important témoignage historique, cf. Lettres de deux amis. Une correspondance entre Penelope S. Delta et Gustave Schlumberger suivie de quelques lettres de Gabriel Millet. Introduction et notes de X. Lefcoparidis. Préface d'André Mirambel. Bibliothèque de l'Institut français d'Athènes, Athènes 1962.

${ }^{8}$ Lettres de deux amis, op.cit. (n. 7), lettres nos : 13, 24, 49, 51-53, $57,59,61,70,73,85$.

${ }^{9}$ Lettres de deux amis, op.cit. (n. 7), lettres nos : 22, 33-34, 37-38, 41-49, 51-53, 57, 59, 63, 69, 75, 77, 78-81, 83-85.

${ }^{10}$ Cf. Lettres de deux amis, op.cit. (n. 7), 146 note 1.
} 


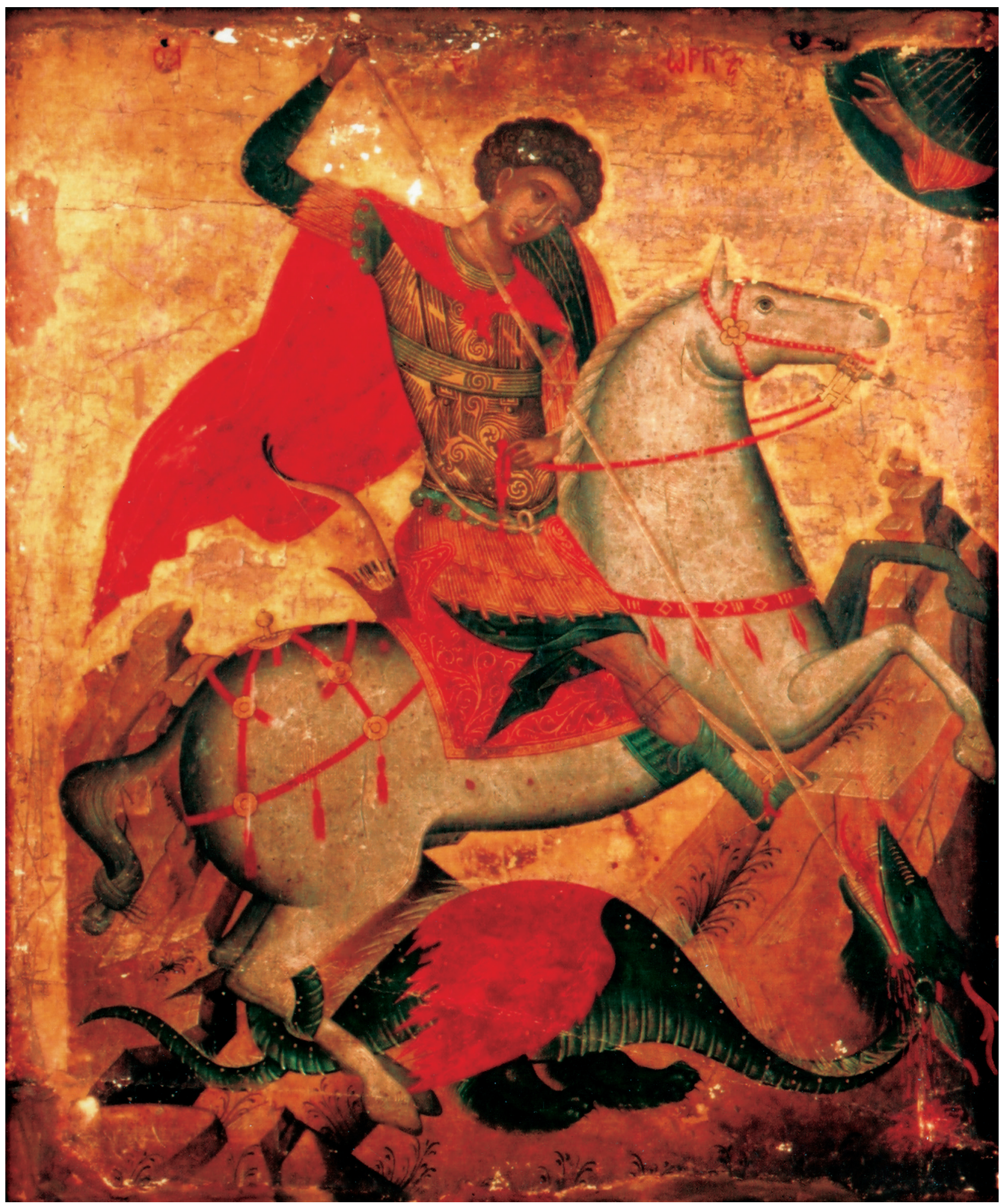

Fig. 1. Strasbourg, musée de l'CEuvre Notre-Dame. Icône de Saint Georges, deuxième moitié du XVe siècle. 
un cheval blanc cabré, tourné vers la droite, aux pieds antérieurs levés, dans un paysage de fond formé de deux collines rocheuses de forme pyramidale qui s'élèvent de part et d'autre de la partie inférieure de la composition. En haut à droite apparaît un segment de ciel avec la main de Dieu qui bénit.

Le saint, tourné de trois quarts vers la droite, a la tête légèrement inclinée en avant et le pied tendu sur l'étrier, il retient les rênes du cheval de sa gauche, et de sa droite levée, attaque de sa lance le dragon qui gît sur le sol, sous le cheval, en relevant sa tête blessée et enlaçant sa queue autour des jambes arrière du cheval. Saint Georges porte une cuirasse métallique finement décorée de chrysographies, formant au centre des rinceaux de forme géométrique $^{11}$. Derrière son épaule gauche apparaît enlacée avec un cordon doré, la partie haute de son bouclier rond, décorée de rayures d'or sur ses deux faces, peintes de couleurs différents : ocre à l'extérieure et bleu foncé dans la partie intérieure. Le cheval blanc peint avec soin, a une queue précieusement nouée et délicatement dessinée. Le harnachement de couleur rouge est riche et soigneusement dessiné avec des attaches dorées, en forme de rosaces sur la tête. Le tapis rouge de la selle est décoré d'un fin dessin doré d'arabesques, finement encadré d'un décor de lettres pseudo-coufiques sur la bordure ${ }^{12}$.

L'iconographie du saint militaire terrassant le dragon bien connue depuis l'époque byzantine ${ }^{13}$, est large-

${ }^{11}$ Cette forme de décor aux rinceaux se répand sur les icônes d'Angelos, parallèlement au simple décor formé de petites plaquettes rectangulaires, cf. N. Chatzidakis, Icons of the Cretan School (15th-16th Century) (cat. exp.), musée Benaki, Athènes 1983, 11, nos 6, 7, 8, 14.

${ }^{12}$ A propos de ce motif sur les icônes italo-crétoises depuis le XVe siècle cf. M. Chatzidakis, "Les débuts de l'école crétoise et

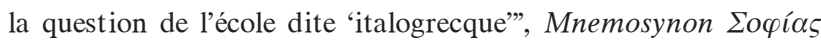

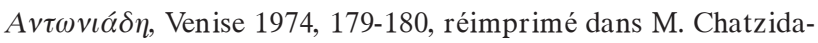
kis, Études sur la peinture post-byzantine, Londres 1976( Variorum Reprints), no IV, p. 180. Voir aussi autres exemples dans N. Chatzidakis, From Candia to Venice, Greek Icons in Italy. 15th-16th Century (cat. exp.), Foundation of Hellenic Culture (Venise, Museo Correr, 1993), Athènes 1993, nos 24, 25, 31, fig. 12.

${ }^{13} \mathrm{C}$. Walter, The Warrior saints in Byzantine art and tradition, Londres 2003, 67-109. (Demetrios et Georges) avec la bibliographie réunie. C. Vanderheyde, "La monture des saints cavaliers dans l'art byzantin”, dans S. Lazaris (dir.), Le cheval, animal de guerre et de loisir dans l'Antiquité et au Moyen Age, Actes des Journées d'étude internationales organisées par l'UMR 7044 ment diffusée sur les icônes crétoises de saint Georges depuis le milieu du XVe siècle, l'exemple le plus important étant celui de l'icône d'Angelos du musée Benaki (Fig. 2) ${ }^{14}$, modèle pour une série d'icônes qui reproduisent fidèlement le sujet au moyen d'un même anthivolon ou poncif ${ }^{15}$, comme l'icône de l'Institut hellénique de Venise ${ }^{16}$, rapprochée de l'art d' Andreas Ritzos, et celle de Corfou (Antivouniotissa), datée vers la fin du XVe siècle (Fig. 3) ${ }^{17}$. Tous les détails de la composition sont reproduits fidèlement, sauf les trois têtes du dragon de l'icône d'Angelos, particularité sans précédent ni suite dans la peinture des icônes. Les ressemblances des vêtements militaires du saint sur ces icônes s'étendent jusqu'aux plus fins détails des chrysographies sur sa cuirasse métallique. Pourtant, à la différence de ces

(Étude des civilisations de l'Antiquité), Strasbourg, 6-7 novembre 2009 (Bibliothèque de l'Antiquité tardive 22), Turnhout 2012, 201-211.

${ }^{14} \mathrm{Ce}$ grand peintre crétois de la première moitié du XVe siècle, est identifié avec le peintre Angelos Akotantos, connu par les documents d'archives. M. Vassilaki, "An Icon of Saint George killing the Dragon by the Painter Angelos: A New Acquisition

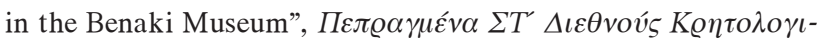

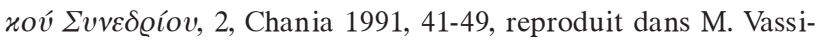
laki, The Painter Angelos and Icon-Painting in Venetian Crete, Londres 2009, 153-169, fig. 8.1. Voir aussi The Hand of Angelos, An Icon-Painter in Venetian Crete (cat. exp.), musée Benaki, M. Vassilaki (dir.), Athènes 2010, no 37, p. 176 (P. Benatou).

${ }^{15}$ N. Chatzidakis, "The contribution of Angelos' works to ctystallizing the iconography of Cretan icons : topics and diffusion of iconographic types (15th-17th c.)" (en grec avec résumé en anglais), Mouseion Benaki, The annual Journal of the Benaki Museum 13-14 (2013-2014), Athènes 2016, 161-183 (pour saint Georges cf. p. 177 et notes 156-166, fig. 22). Pour l'usage des "an-

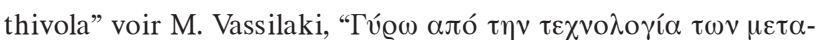

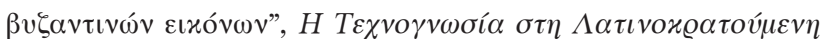

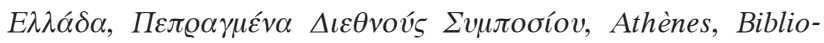
thèque Gennadios, 1997, Athènes 2000, p. 195-206 (article publié ultérieurement en anglais sous le titre: "On the technology of post-byzantine icons", dans Vassilaki, The Painter Angelos, op.cit., p. 333-344).

${ }^{16}$ M. Chatzidakis, Icônes de Saint-Georges des Grecs et de la collection de l'Institut (Bibliothèque de l'Institut hellénique d'études byzantines et post-byzantines de Venise 1), Venise 1962, 119, no 100 , pl. 58.

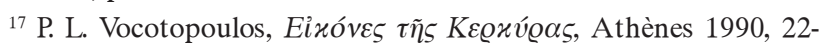
24 , no 10, fig. 12; voir autres exemples dans Vassilaki, "An Icon of Saint George” op.cit. (n. 14), 46-49, pl. 31b, 32a-b, 33. 


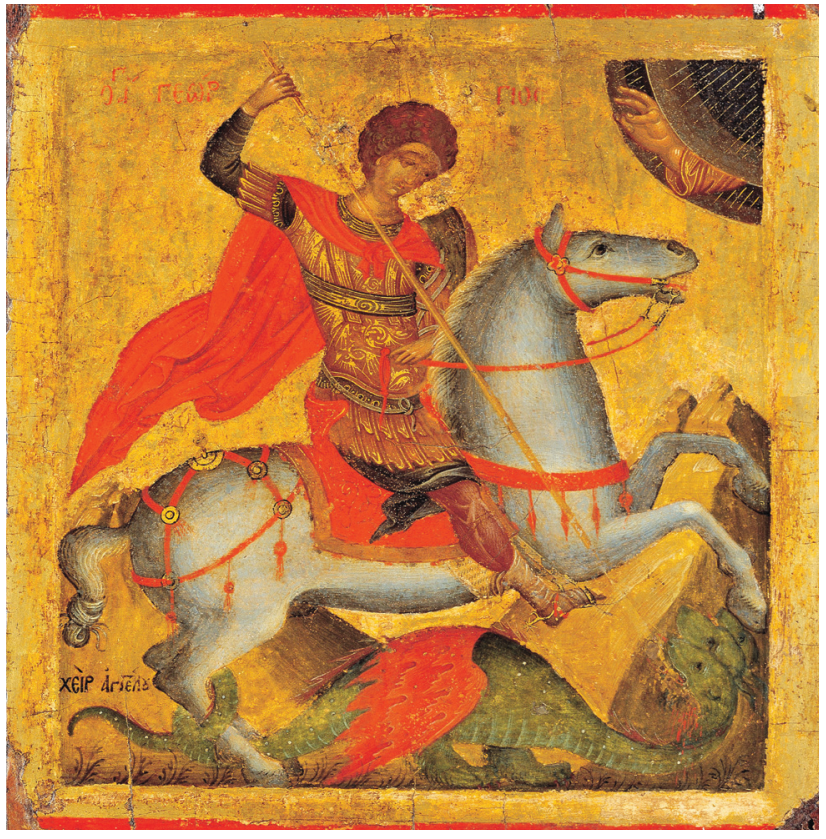

Fig. 2. Athènes, musée Benaki. Icône de Saint Georges, peintre Angelos, première moitié du XVe siècle.

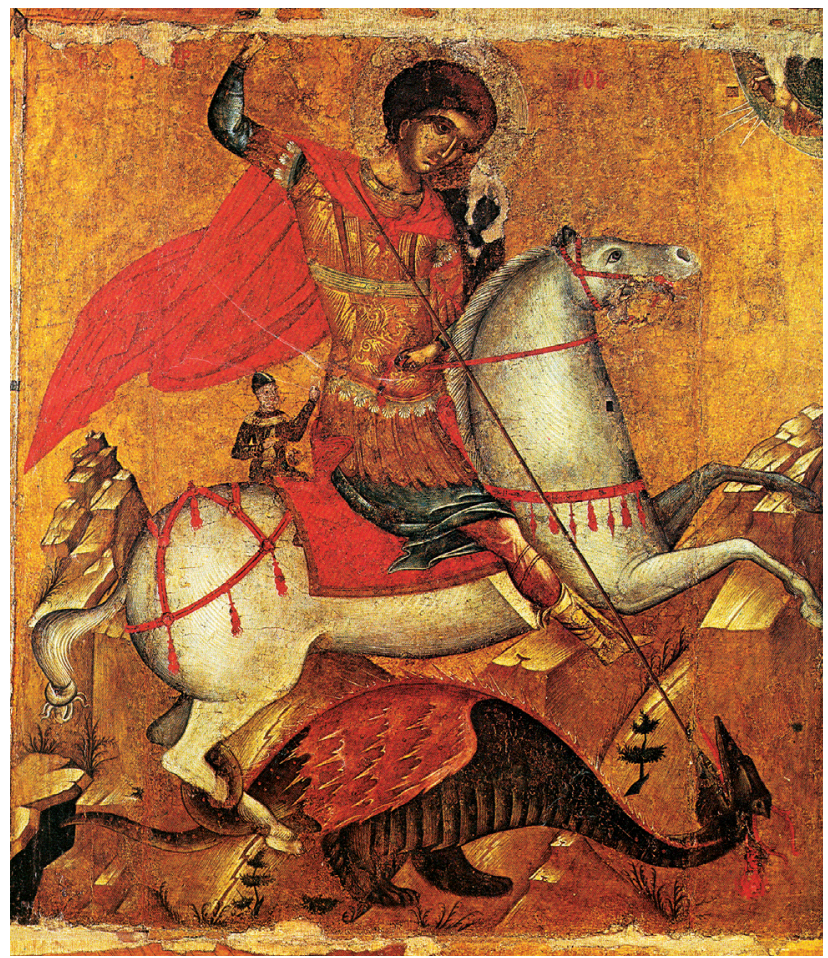

Fig. 3. Corfou, musée de l'Antivouniotissa. Icône de Saint Georges, c. 1500. icônes, l'armement du saint sur l'icône de Strasbourg est enrichi par un arc accroché ensemble avec un carquois rempli de flèches, sur la selle du cheval derrière le dos du saint. Finement dessinés, ces éléments apparaissent également sur d'autres représentations où le saint cavalier est représenté en parade, comme sur les fresques de l'église de Prassès Kydonias en Crète (première moitié du XVe siècle) et l'icône de Lithines, en Crète (milieu XVe siècle $)^{18}$, ainsi que sur une autre icône du Musée de la ville de la Canée, datée vers $1500^{19}$, où saint Georges est représenté terrassant le dragon, comme sur notre icône. Nous retrouvons le même équipement du saint sur une série d'icônes où l'iconographie est enrichie par certains détails narratifs, comme la princesse libérée devant la tour du palais et le jeune esclave libéré monté sur le dos du cheval, comme sur l'icône d'une collection privée de Venise datée vers le milieu du XVe siècle ${ }^{20}$ (Fig. 4). Une autre icône du peintre crétois Zacharias Tzankaropou$\operatorname{los}^{21}(108 \times 91 \mathrm{~cm})$, signée et datée de 1635 , sur le templon de l'église de saint Georges Aphentis au quartier de Hagios Loukas d'Artémonas, à Siphnos ${ }^{22}$ (Fig. 5), offre un exemple représentatif de la diffusion du même modèle parmi les peintres crétois du XVIIe siècle. En plus, il est intéressant de noter que la formule iconographique simple du saint cavalier tuant le dragon se trouve également sculptée en relief sur une plaque de marbre datée

${ }^{18}$ N. Chatzidakis, "Saint Georges on a Horseback. A Fifteenth Century Icon in the Benaki Museum”, $\Theta v \mu i ́ \alpha \mu \alpha \sigma \tau \eta \mu v \eta \mu \eta \tau \eta \varsigma \Lambda \alpha-$

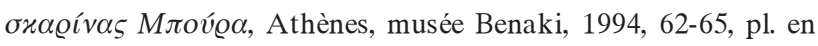
couleurs VI-VII, et pl. 30-32, nos 2-11 fig. 1-11. Pour ce type, voir aussi N. Chatzidakis, "Icône de saint Georges au musée du Louvre", op.cit. (n. 1), 2015, 63-67, fig. 7.

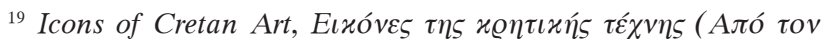

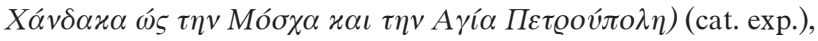
dir. M. Borboudakis, Hérakleion 1993, no 173, p. 526 (M. Borboudakis). Sur une zone supérieure apparaît la Sainte Trinité.

${ }^{20}$ N. Chatzidakis, From Candia to Venice, op.cit. (n. 12), 1993, 36-39, no 4.

21 "XЕIP ZAXAPIOY TZAKAPOПOY $\Lambda$

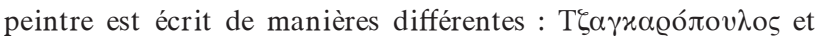

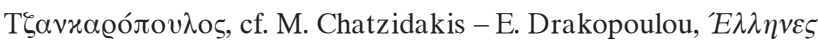

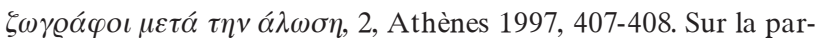
tie supérieure de l'icône on voit la Décapitation du saint, à petite échelle, dépeinte au début du XXe siècle.

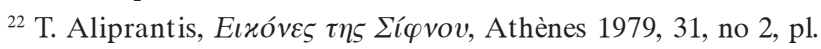
17,18 et couverture. Chatzidakis - Drakopoulou, ${ }^{2} E \lambda \lambda \eta v \varepsilon \varsigma \xi \omega-$

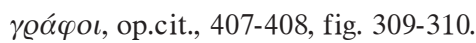




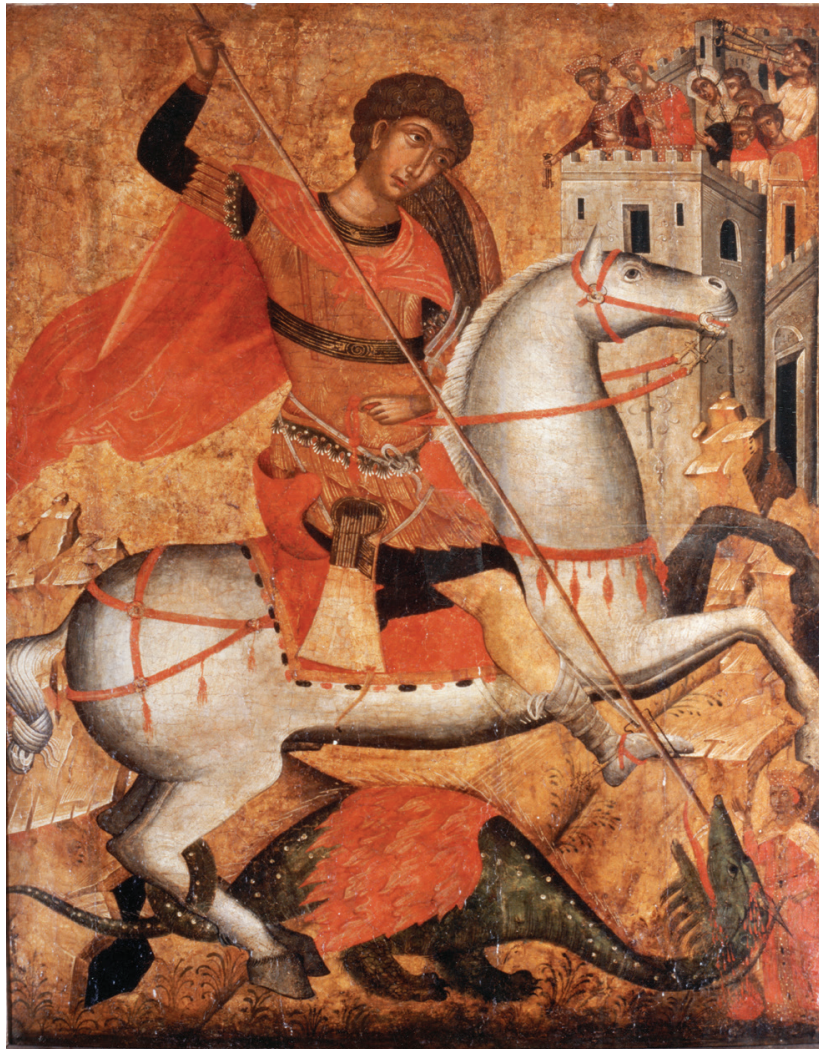

Fig. 4. Venise, collection privée. Icône de Saint Georges, milieu du XVe siècle.

de 1630, incrustée sur la façade au-dessus de la porte d'entrée de la même église (Fig. 6) ${ }^{23}$.

Il faut enfin signaler que le cheval sur l'icône de Strasbourg, alors qu'il est bien ajusté en largeur dans l'encadrement, semble pourtant légèrement décroché de l'espace physique, puisque ses pieds arrière ne sont pas disposés conformément aux pentes du sol. Bien que sur la plupart des exemples déjà mentionnés le cheval soit très attentivement adapté au paysage, comme sur l'icône d'Angelos du musée Benaki (Fig. 2), il y a d'autres exemples de haute qualité comme les icônes de Corfou de la fin du XVe siècle (Fig. 3) et de la collection privée de Venise (Fig. 4) marquées par une négligence à ce sujet, observée également sur l'icône de Tzankaropoulos de Siphnos (1635) (Fig. 5).

\footnotetext{
${ }^{23}$ Inédite. La date de 1630, correspond apparemment à la date de la construction de l'église.
}

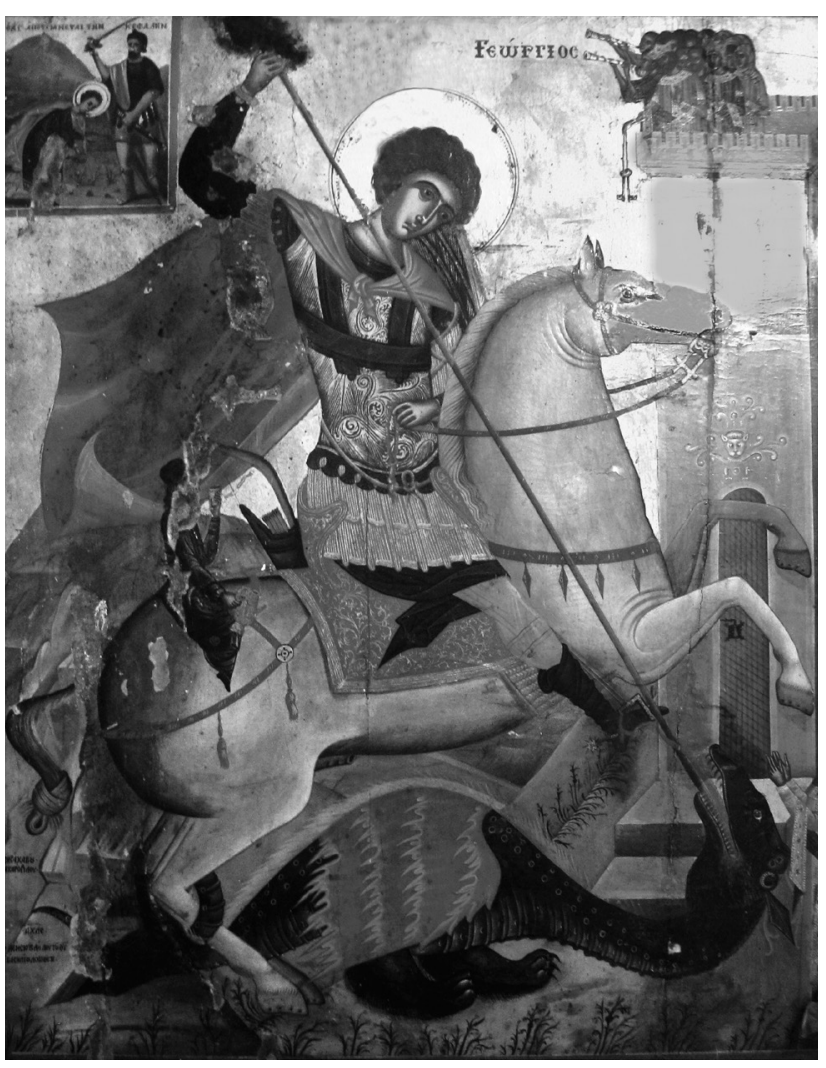

Fig. 5. Siphnos, Saint-Georges Aphentis. Icône de saint Georges, peintre Zacharias Tzankaropoulos, 1635.

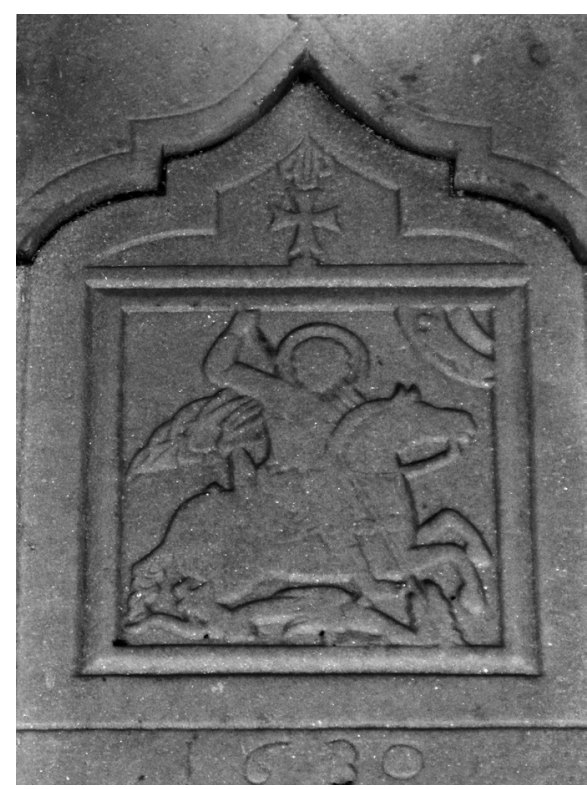

Fig. 6. Siphnos, Saint-Georges Aphentis. Icône en marbre, saint Georges, 1630. 
Après cet examen de l'iconographie, nous constatons que l'icône de saint Georges du Musée de l'Euvre NotreDame de Strasbourg, reproduit, malgré quelques variations, un type cristallisé dans la peinture des icônes crétoises du XVe siècle, comme les icônes de Corfou (Fig. 3), de l'Institut hellénique et de la collection privée de Venise (Fig. 4), qui suivent fidèlement le plus ancien exemple de la série, repéré sur l'icône d'Angelos (Fig. 2). En plus, on peut noter que par les qualités de son style l'icône de Strasbourg (Fig. 1) se rapproche davantage de l'art de ce peintre : l'attitude du cheval blanc et le rendu volumineux de son corps, le manteau rouge du saint qui flotte ondulé en arrière, en formant exactement les mêmes larges plis, le dessin minutieux des chrysographies sur les parties métalliques du costume militaire, la cuirasse et son bouclier, ainsi que le harnachement du cheval, ont un traitement identique. Les ressemblances s'étendent au traitement du modelé de la chair par des fines touches sombres, ainsi qu'au délicat dessin des traits du visage sévère et attristé. L'ensemble de ces remarques permettent l'attribution de l'icône de Strasbourg à un peintre crétois de la deuxième moitié du XVe siècle, travaillant suivant le style et d'après les modèles du peintre Angelos.

En conclusion, nous devons remarquer que l'appartenance de l'icône de Strasbourg au legs Schlumberger offre un témoignage éloquent sur l'étendue des intérêts de ce grand collectionneur et dévoile un goût raffiné pour la peinture des icônes, à une époque où les grands peintres crétois du XVe siècle étaient inconnus des spécialistes ${ }^{24}$. Par son intuition aiguë, il a su enrichir sa collection d'une belle icône crétoise de la deuxième moitié du XVe siècle, acquise très probablement au cours de ses voyages en Grèce ou à Venise ${ }^{25}$.

\footnotetext{
${ }^{24}$ L'art des icônes crétoises du XVe siècle a été réapprécié et correctement daté seulement après la publication des documents d'archives et les études de M. Chatzidakis parues dans la décennie de 1970 ; voir la bibliographie réunie entre autres dans N. Chatzidakis, From Candia to Venice, op.cit. (n. 12), 3-25 et notes.

${ }^{25}$ Voir plus haut p. 227-228 et notes 4-10. Nous savons que le grand collectionneur russe N. Lichačev (1862-1936) avait acquis une grande part des icônes crétoises de sa collection auprès des antiquaires de Venise. Y. Piatnitsky (dir.), Iz kollektkcij akademika N. P. Lihaceva, Saint-Pétersbourg 1993.
}

\section{L’icône de la Déisis}

L'icône de la Déisis $(48,5 \times 37,5 \mathrm{~cm})^{26}$ de provenance inconnue, est préservée en bonne condition, ayant subi dans le passé une rénovation du fond doré (Fig. 7, 8). Sur la partie supérieure du fond doré, repeint à une époque postérieure mais inconnue, on voit des inscriptions en caractères rouges gréco-latins, en partie abîmées, qui accompagnent les trois personnages reproduisant très probablement les inscriptions originales. A côté de la Vierge on distingue les caractères latins : $M[A] T[E R]$ $D O[M I N E]$. A gauche et à droite du Christ deux fois le même mot: J[E]C[UC]. A côté de saint Jean le Précurseur on reconnaît les caractères grecs : $O A(\Gamma I O C)$ $I \Omega(A N N H C)$ O ПPO $\triangle P O M O C$. Une autre inscription en lettres grecques, en rouge, apparaît dans la croix qui décore le nimbe doré du Christ : $\omega(\text { sic })^{27} \omega \mathrm{N}$.

La composition comprend le Christ debout au centre, en position frontale, tenant un évangile fermé et bénissant de sa droite avec la paume tournée vers le spectateur; il porte un himation de couleur bleu foncée qui enveloppe son corps imposant par des plis larges et couvre en partie un chiton long de couleur rouge lim-

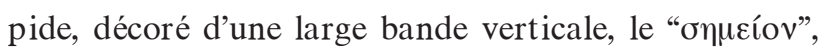
décoré de rayures d'or. La surface de la couverture de son évangile est également décorée de rayures dorées. Il repose sur un socle en bois rectangulaire, décoré de libres rinceaux en chrysographie. Il est flanqué des figures élancées de la Vierge à gauche et de saint Jean le Précurseur à droite, debout et tournés de trois quarts, en supplication vers lui, en tenant des rouleaux dépliés vers le bas. La Vierge porte un maphorion rouge cerise limpide qui couvre par des plis amples une robe bleue foncée ; son liseré est décoré d'une large bande ornée d'un fin dessin de lettres pseudo-couphiques en chrysographie, accompagnée d'une frange également en chrysographie. Saint Jean porte un himation de couleur beigeocre qui couvre amplement un chiton long de couleur rouge clair. Les rouleaux sont rendus avec un grand soin pour rendre le le mouvement en serpentine, alors que les lettres grecques des inscriptions, bien calligraphiées en noir, se dégagent sur le fond blanc; le peintre utilise

\footnotetext{
${ }^{26}$ No Inv. 22.995.0.660.1.

${ }^{27}$ Il s'agit d'une faute d'orthographe pour l'article masculin "O", rarement rencontrée.
} 


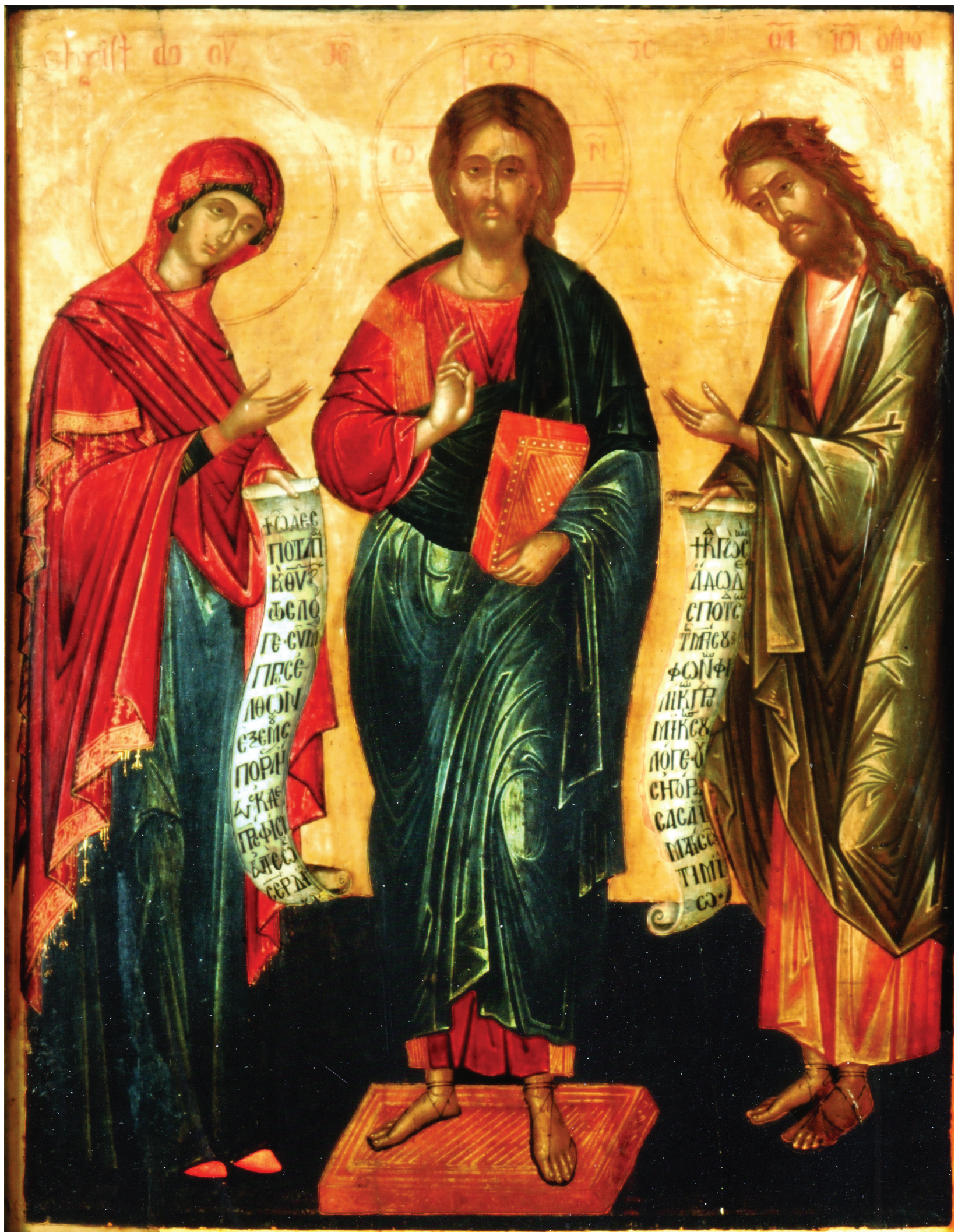

Fig. 7. Strasbourg, musée de l'QEuvre Notre-Dame. Icône de la Déisis, milieu du XVIe siècle. 

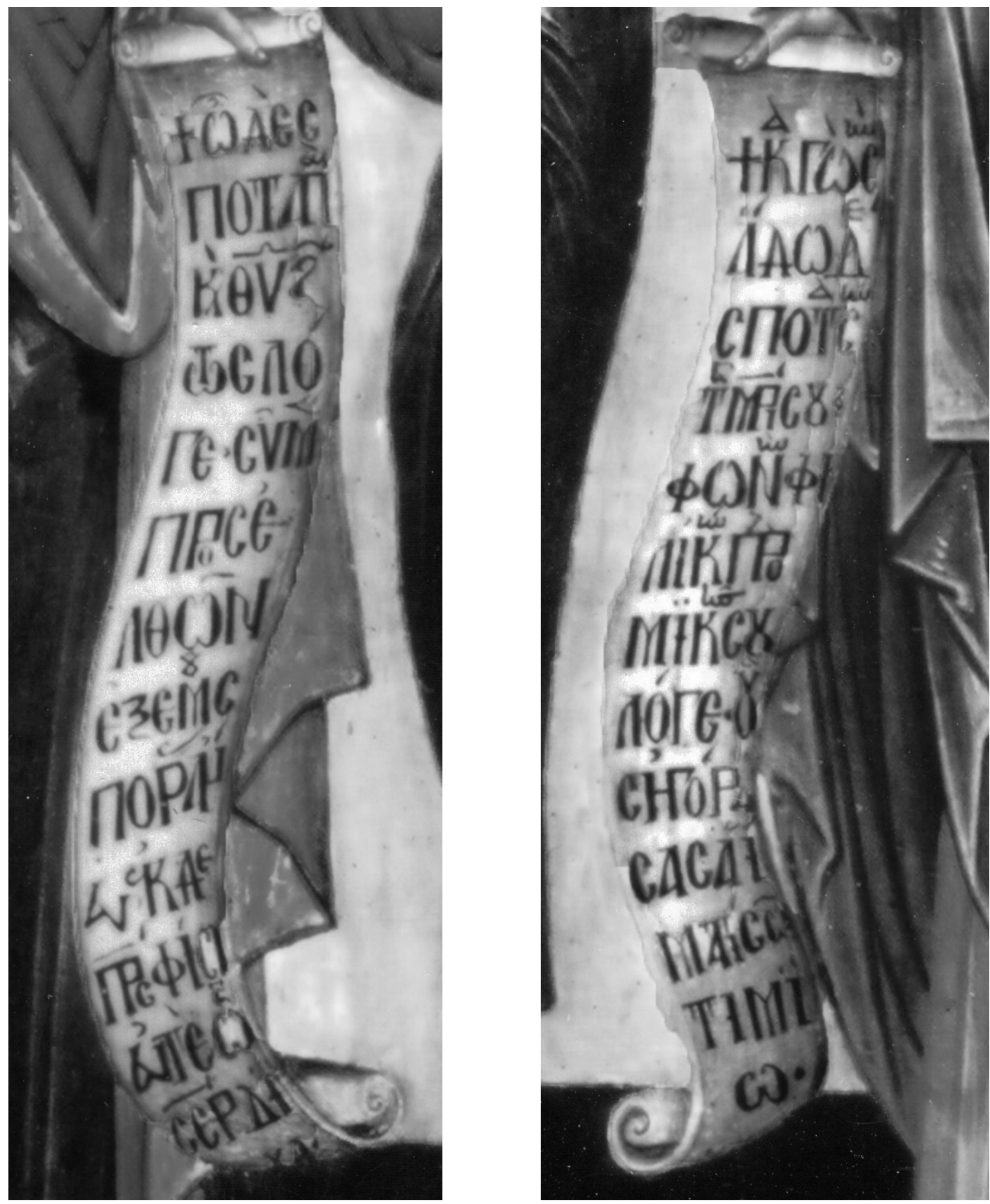

Fig. 8. Strasbourg, musée de l' Euvre Notre-Dame. Icône de la Déisis (détail).

avec élégance des accents, des esprits, des ligatures et quelques lettres en minuscule, intercalées discrètement dans le texte, alors que le début de la prière est précédé d'une croix (Fig. 8).

Vierge : $+\Omega \triangle E C / \Pi O T A \Pi \alpha \iota / K(A I) \Theta(E O) Y Z(\Omega N)$ $/ T \Omega C \Lambda O / \Gamma E \cdot C Y M / \Pi P O C E / \Lambda \Theta \Omega N / E \Xi E M(O Y)$ C/ПOPAC AN/EY EK $\triangle E / \Pi(A T) P(O) C$ ФICI P/ EYT $(\sigma) E \Omega \Sigma / C(\Omega T) E P \triangle I / X A$.
Jean : + KAГ $C v v / A \Delta \Omega \Delta E / C \Pi O T A C \omega(\tau \varepsilon \varrho) / T H$ $M[H T] P I C O Y / \Phi \Omega N \eta \Phi I / \Lambda I K \eta ~ \Pi P O /[\triangle P O] M I K \eta$

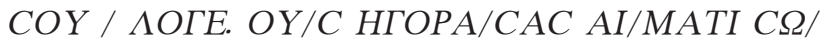
$T I M I / \Omega$.

\section{Iconographie}

L'origine du sujet de la Vierge intercédant en prière pour le salut de l'humanité, remonte au XIIe siècle, sur des 
icônes hautement vénérés à Constantinople, comme la Vierge Hagiossoritissa ou Paraklesis (en prière) ${ }^{28}$. Dans des représentations indépendantes elle est tournée de trois quarts, tenant un rouleau déplié avec le texte de la prière de la Déisis, comme sur les plus anciens exemples connus de la mosaïque de la Martorana et des icônes du Sinaï et de Spolète des XIIe-XIIIe siècles ${ }^{29}$. Encore, on peut noter l'origine byzantine du sujet autonome de saint Jean Précurseur ailé, dans le désert, debout tourné de trois quarts en prière et tenant un rouleau déplié avec un texte de prière ${ }^{30}$, sujet répandu par la suite dans la peinture crétoise au moyen des poncifs des icônes du peintre Angelos au musée Byzantin d'Athènes et au Musée de Malines en Belgique ${ }^{31}$.

Le sujet de la Déisis où la Vierge et saint Jean, tournés en prière vers le Christ, tiennent des rouleaux avec inscriptions, est assez répandu sur les fresques qui décorent la conque de l'abside ou bien d'autres parties des

${ }^{28}$ C. Walter, "Two Notes on the Deisis", REB 26 (1968), 311- 336. S. Der Nersessian, "Two Images of the Virgin”, DOP 14 (1960), 77-78, fig. 6-13. M. Andaloro, "Note sui temi iconografici della Deesis e della Haghiosoritissa”, Rivista dell'Istituto Nazionale d'Archeologia e Storia dell'Arte 17 (1970), 85 et s., p. 129 et s. Voir

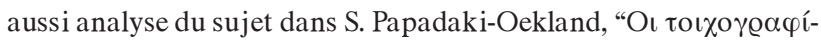

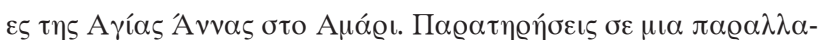

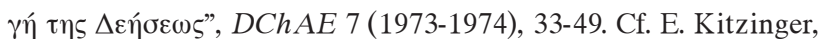
The Mosaics of Saint Mary's of the Admiral in Palermo, D.O. Research Library, Washington, D.C. 1990, 199-205.

${ }^{29}$ Kitzinger, The Mosaics of Saint Mary's, op.cit., 199-205, pl. XXII. G. et M. Sotiriou, Icônes du Monastère de Sinaï (en grec), II, Athènes 1958, fig. 173. M. Bonfioli, "L'icona del Duomo di Spoleto, Nuovi contributi alla sua storia", Spoletium 33 (décembre 1988), 17-25, fig. 2, 3.

${ }^{30}$ Lafontaine-Dosogne, "Une icône d'Angélos et l'iconographie de Saint-Jean Baptiste ailé”, Bulletin des Musées Royaux d'Art et d'Histoire, 48e année (1976), Bruxelles 1978, 142-143. Voir a ussi exemples dans N. Chatzidakis, Icons. The Velimezis Collection, Catalogue raisonné, Benaki Museum, Athènes1998, no 10, p. 116-121. ${ }^{31}$ N. Chatzidakis, "The Legacy of the Painter Angelos", The Hand of Angelos, op.cit. (n. 14), 124-133. Pour l'icône du musée Byzantin (provenant de l'ancienne collection E. Hatzidakis), voir et M.

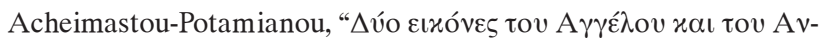

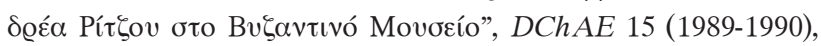
105-118, fig. 1-4. Pour l'icône de Malines, voir J. Lafontaine-Dosogne, "Une icône d'Angélos" op.cit. (n. 31), 121-143. The Hand of Angelos, op.cit. (n. 14), no 38, p.178-179 (A. Lymberopoulou). Voir aussi autres exemples réunis dans N. Chatzidakis, "The contribution of Angelos' works", op.cit. (n. 15), 171, fig. 14 et notes 101-112.

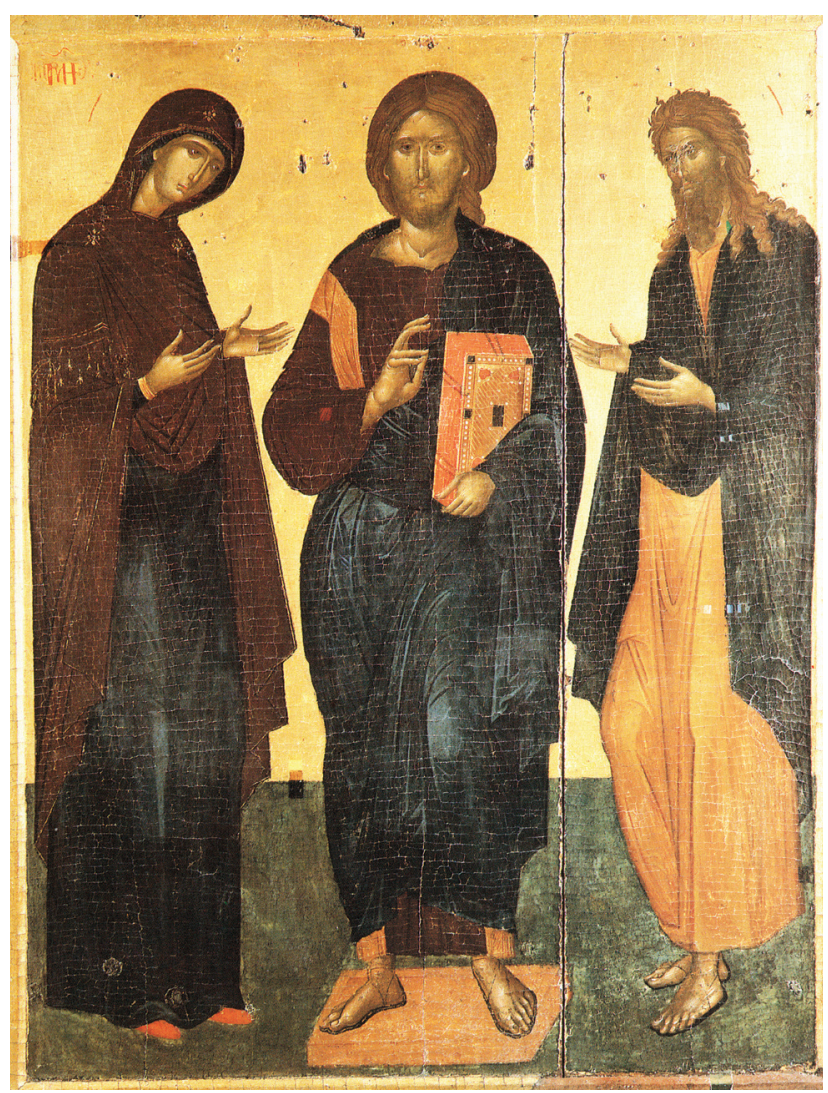

Fig. 9. Crète, Moni Odigitrias. Icône de la Déisis, c.1400.

églises byzantines, dès la fin du XIIe siècle, dans différentes régions de l'empire byzantin, en Crète comme à Sainte-Anne d'Amari, ainsi qu'à Rhodes, à Kastoria et ailleurs $^{32}$.

Une iconographie, avec les trois figures saintes en pied, mais sans rouleaux dépliés, peut être repérée sur une icône du monastère de Sinaï du XIIe siècle, où la figure de saint Nicolas est ajoutée à gauche de la composition $^{33}$. Le modèle sinaïtique se retrouve sur une icône crétoise de qualité, datée vers 1400 de la Moni Hodigitrias en Crète $(115 \times 85,5 \mathrm{~cm})$, où le Christ est placé

\footnotetext{
${ }^{32}$ Voir plusieurs exemples réunis dans S. Papadaki-Oekland, "Oı

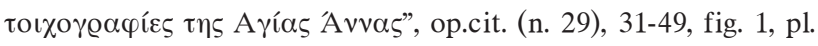
8,10 . Voir aussi plusieurs exemples cités dans S. Maderakis, " $\mathrm{H}$

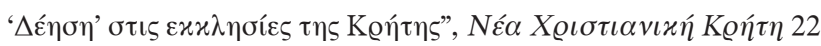
(Réthymnon 2003), 10-110, en particulier p. 34-60, 89-100, fig. $28,30,39$. Dans tous les cas le texte des inscriptions des rouleaux diffère essentiellement de celui de notre icône.

${ }^{33}$ Sotiriou, Icônes, op.cit. (n. 29), no 48.
} 


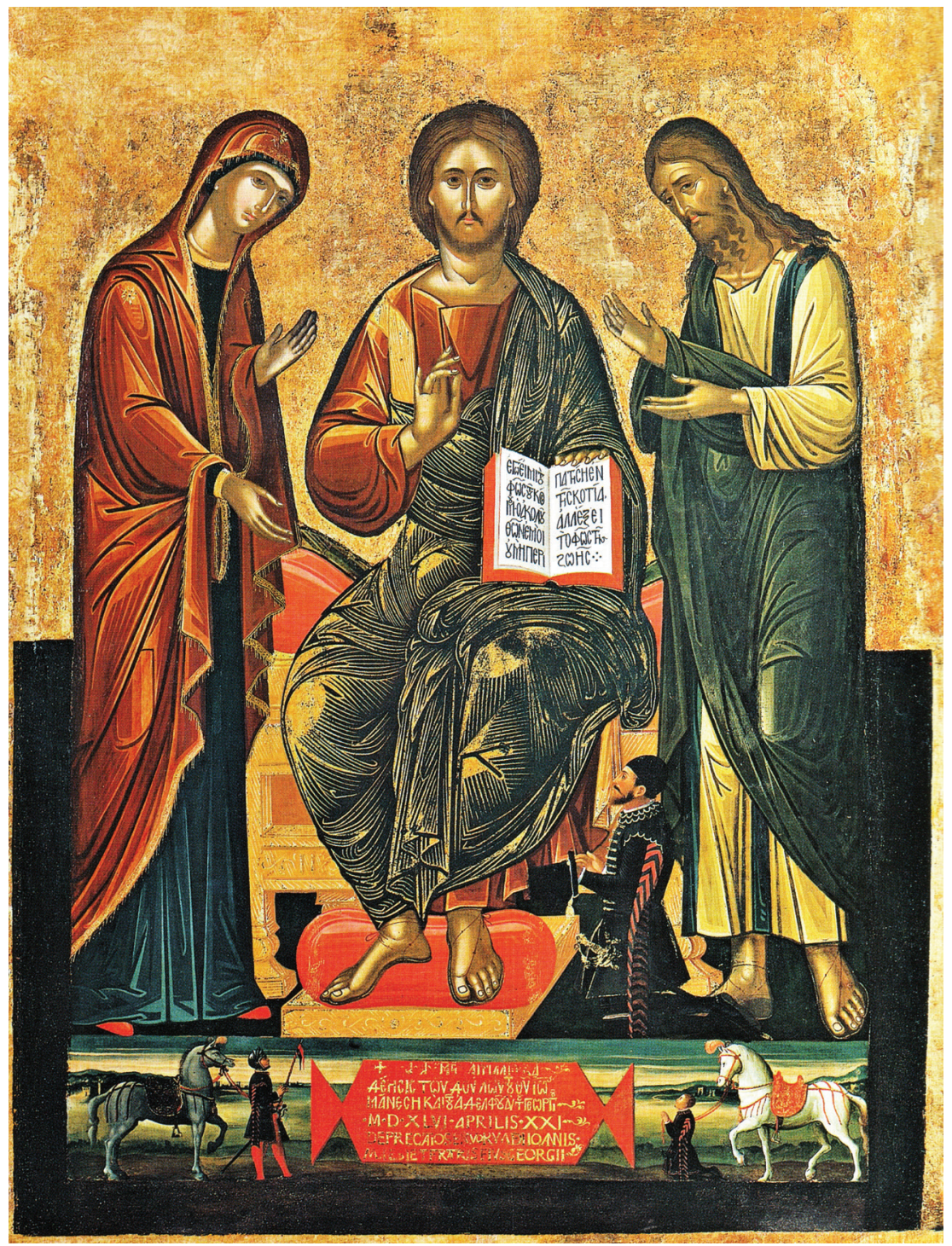

Fig. 10. Venise, église de Saint-Georges des Grecs. Icône de la Déisis, donateurs frères Manessis, Venise 1546.

sur un piédestal comme sur notre icône ${ }^{34}$ (Fig. 9). Sur une autre icône de la Déisis, datant du début du XVIe siècle, au musée de Princeton, nous trouvons encore un exemple de cette iconographie ${ }^{35}$. Des variantes de ce type

${ }^{34}$ Icons of Cretan Art, op.cit. (n. 19), no 116, p. $492-493$ (M. Borboudakis).

${ }^{35}$ Dimensions : $25,5 \times 21 \mathrm{~cm}$. D. Mouriki, "A Deisis Icon in the Art Museum", Record of the Art Museum, Princeton University 27/1 (1968), 13-28. Holy Image, Holy Space, Icons and Frescoes from Greece, Byzantine Museum of Athens - The Walters Art sont également connues sur une autre icône du Sinaï du milieu du XVe siècle, où saint Jean est remplacé par saint Phanourios, alors que le Christ est placé sur un socle rectangulaire comme sur notre icône ${ }^{36}$; sur un autre exemple de la fin du XVe siècle, dans l'annexe sinaïtique

Gallery, Baltimore, Maryland, Athènes 1988, no 67, p. 152, 223 (N. Teteriatnikov).

${ }^{36}$ Dimensions : $24,4 \times 16,9 \mathrm{~cm}$. N. Drandakis dans Sinaï. The Treasures of the Monastery, Athènes 1990, 127, fig. 77, attribuée au peintre Angelos. 


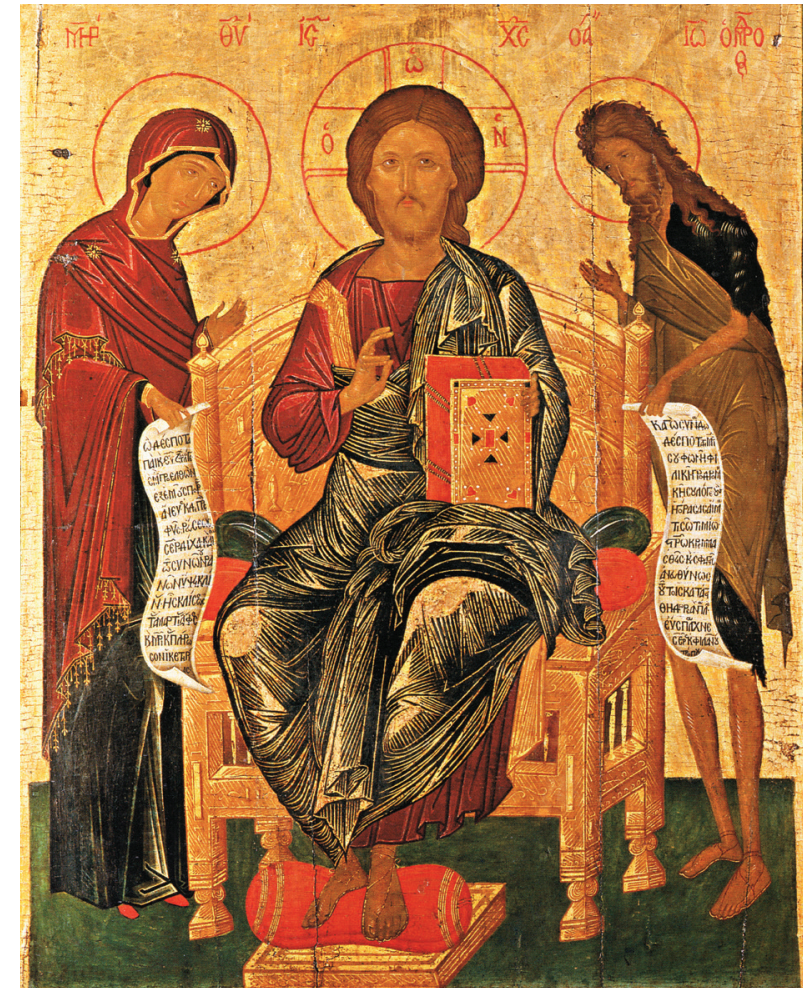

Fig. 11. Athènes, musée Byzantin. Icône de la Déisis, c.1500.

d'Héraklion, Crète, saint Jean est remplacé par saint $\mathrm{Ni}$ colas et le Christ debout repose sur un coussin rouge ${ }^{37}$. Le sujet se répand au cours des XVIe-XVIIe siècles sur les icônes en Crète et dans les îles ioniennes, comme sur une icône de Léos Moschos, datée de 1649, de la Collection Vélimézis, où à la place de saint Jean est figuré saint Georges tenant dans sa main sa tête décapitée ${ }^{38}$.

Une série d'icônes de la Déisis, datées du XVe au XVIIe siècle, peut également s'apparenter au type iconographique de notre icône avec une variation importante : le Christ au centre de la composition est représenté trônant, et les figures saintes posent en prière sans tenir de rouleaux, suivant une formule largement diffusée, au moyen des modèles (anthivolon) d'après une série d'œuvres du peintre Angélos, comme sur l'icône du musée Canellopoulos ${ }^{39}$. Ce même type est repris sur

\footnotetext{
${ }^{37}$ Dimensions : $97 \times 80 \mathrm{~cm}$. Drandakis, Sinä̈, op.cit. (n. 36), 132133, 210, fig. 84 , peintre crétois de la fin du XVe siècle.

${ }^{38}$ N. Chatzidakis, Velimezis Collection, op.cit. (n. 30), 300-305, no 35.

${ }^{39}$ Voir N. Chatzidakis, "The Deisis by the Painter Angelos in the
}

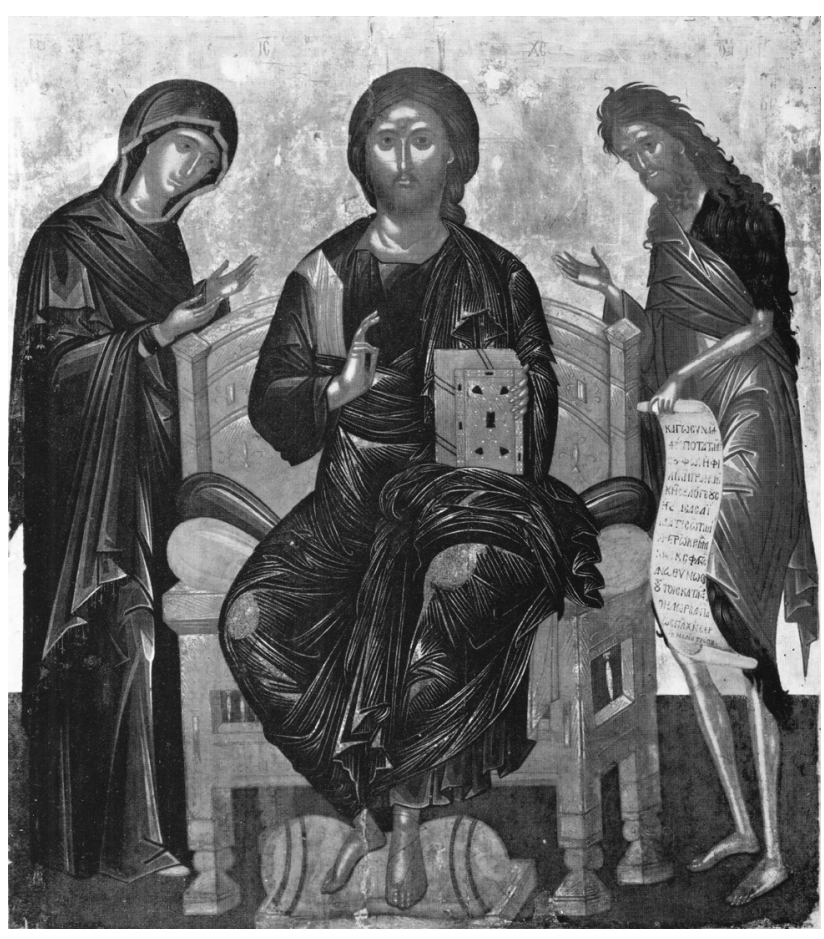

Fig. 12. Vienne, Kunsthistorisches Museum. Icône de la Déisis, c. 1500 .

une grande icône de la Déisis, à l'église Saint-Georges des Grecs de Venise (Fig. 10) ${ }^{40}$, où le Christ trônant tient l'évangile ouvert avec une inscription ${ }^{41}$, alors que la Vierge vêtue d'un maphorion de couleur rouge-cerise sur une robe bleu foncé et saint Jean vêtu d'un chiton et d'un himation longs, sont tournés en prière vers le Christ. Sur une zone étroite de la partie inférieure de l'icône, les portraits des donateurs, les frères Manessis,

Canellopoulos Museum, and the Use of its Anthivolon in the Fifteenth Century”, DChAE 27 (2006), 283-296 (en grec et un résumé en anglais). N. Chatzidakis, "The Legacy of the painter Angelos", dans The Hand of Angelos, op.cit. (n. 14), 124-133. N. Chatzidakis, "The contribution of Angelos' works" op.cit. (n. 15), 164-165 et notes 37-50, fig. 2, 5, 6 .

${ }^{40}$ Dimensions : $119 \times 89 \mathrm{~cm}$. Chatzidakis, Icônes de Venise, op.cit. (n. 16), 21-22, no 8, pl. 6, 8. Voir aussi, Arte Bizantina e Postbizantina. Museo di Icone dell'Istituto Ellenico di Venezia (textes M. Kazanaki, préface Ch. Maltezou), Venise 2005, 224-225, fig. couleur à la page 224.

41 "ЕГ $\Omega$ EIMI TO $\Phi \Omega C$ TOY KOCMOY. O AKO $\Lambda O Y \Theta \Omega N$ EMOI OY MH ПЕPIПATHCH EN TH CKOTIA, A $\Lambda \Lambda^{\prime}$ EEEI TO $\Phi \Omega C$ THC Z $\Omega H C "$. Chatzidakis, Icônes de Venise, op.cit. (n. 16), 21 


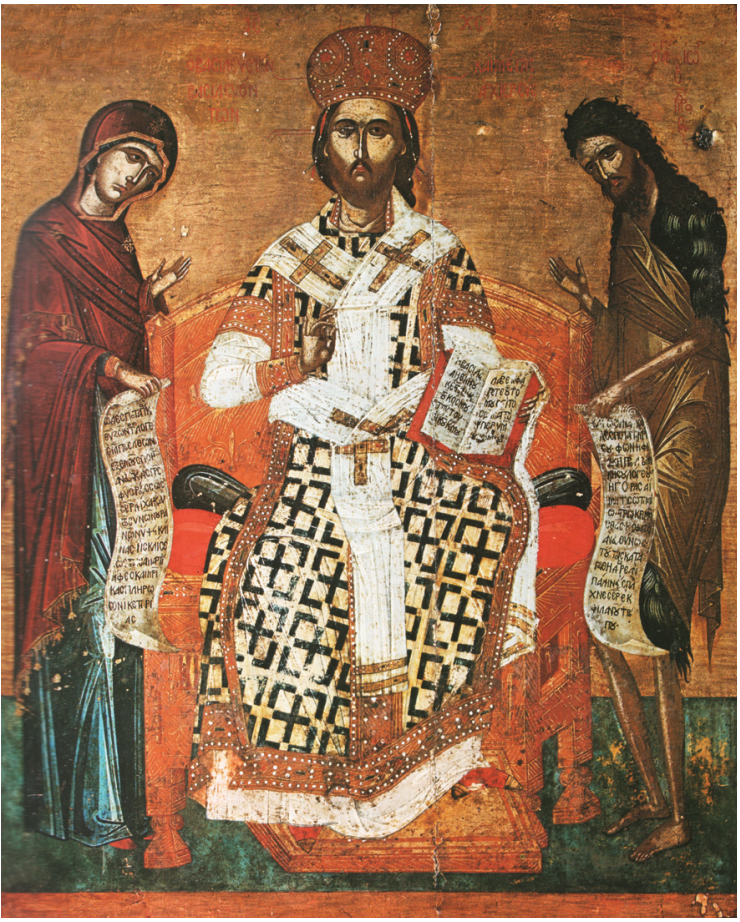

Fig. 13. Patmos. Icône de la Déisis c. 1600-1610.

figurés en costume de soldats vénitiens, flanquent une belle inscription bilingue, en grec et en latin, préservant leurs noms et la date de 1546.

La Vierge et saint Jean, tenant de longs rouleaux dépliés avec les textes d'une prière d'intercession ${ }^{42}$ comme sur notre icône sont repérés sur une autre série d'icônes crétoises de la fin du XVe jusqu'au XVIIe siècle, suivant pourtant une version qui présente deux remarquables différences : (a) Le Christ est représenté toujours assis sur le trône. (b) Saint Jean ne porte pas le costume du prêcheur comme sur l'icône de Strasbourg, mais il est montré en tant qu'ascète du désert, amaigri et revêtu seulement d'une mélote et d'un himation court qui laisse ses jambes découvertes.

L'exemple le plus ancien est repéré sur une icône despotique au musée Byzantin d'Athènes datée vers $1500^{43}$

\footnotetext{
${ }^{42}$ Le texte des inscriptions sera examiné plus loin.

${ }^{43} \mathrm{~K}$. Kreidl-Papadopoulos, "Die Ikonen im Kunsthistorisches Museum in Wien”, Jahrbuch der Kunsthistorischen Sammlungen in Wien 66 (1970), 95, fig. 60. M. Acheimastou-Potamianou, Icons of the Byzantine Museum of Athens, Athènes 1998, 152-153, no 44.
}

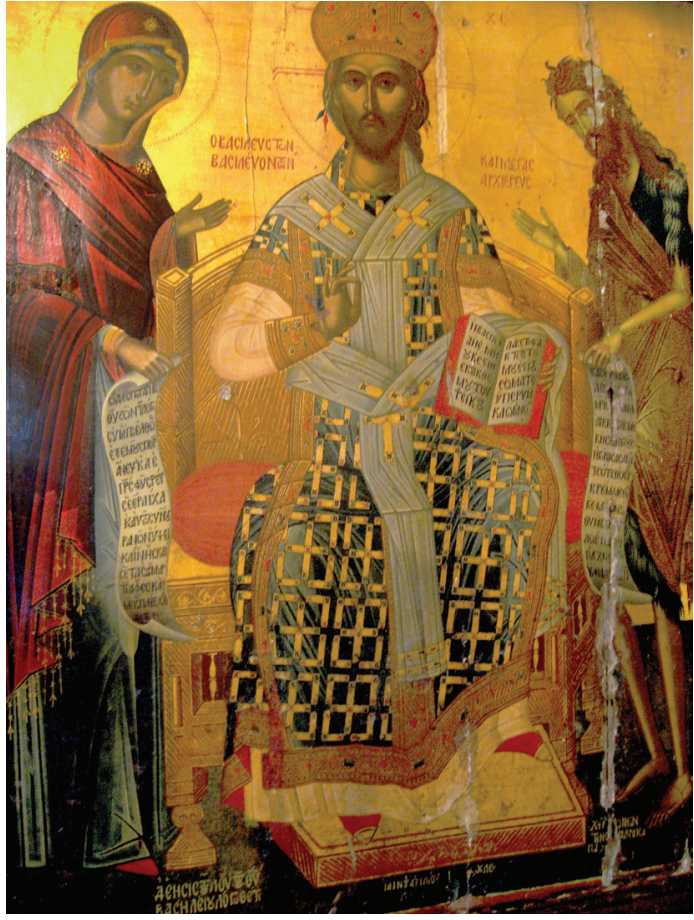

Fig. 14. Siphnos. Saint-Georges Aphentis. Icône de la Déisis, Constantin Paléokappas 1635.

(No Inv. T.305, 94×75 cm) (Fig. 11). Le Christ portant le chiton et l'himation est assis frontalement sur un trône de bois, décoré de rayures d'or. Il pose ses pieds sur un coussin rouge et il tient de sa main gauche un évangile fermé. La Vierge vêtue d'un maphorion rouge brique qui enveloppe son corps, tournée de trois quarts vers le Christ, tient un rouleau déplié avec le texte de la prière de l'intercession.

Saint Jean de l'autre côté, figure amaigrie, vêtu d'une mélote et d'un himation courts, tourné de trois quarts vers le Christ, tient un rouleau déplié avec une longue inscription de prière. Sur une autre icône despotique de la Déisis de la même époque, au Kunsthistorisches Museum de Vienne (no. d'inv. 6549, 81×71,2 cm) ) $^{44}$, la Vierge est tournée avec les deux mains en prière vers le Christ, alors que seulement Jean tient le rouleau déplié avec le texte de la prière (Fig. 12).

A ces exemples nous pouvons ajouter d'autres variantes de la Déisis avec la Vierge et saint Jean tenant des

${ }^{44}$ Kreidl- Papadopoulos, Ikonen, op.cit. (n. 43), 66, 95, no 3, fig. 42 (fin XVe siècle). 


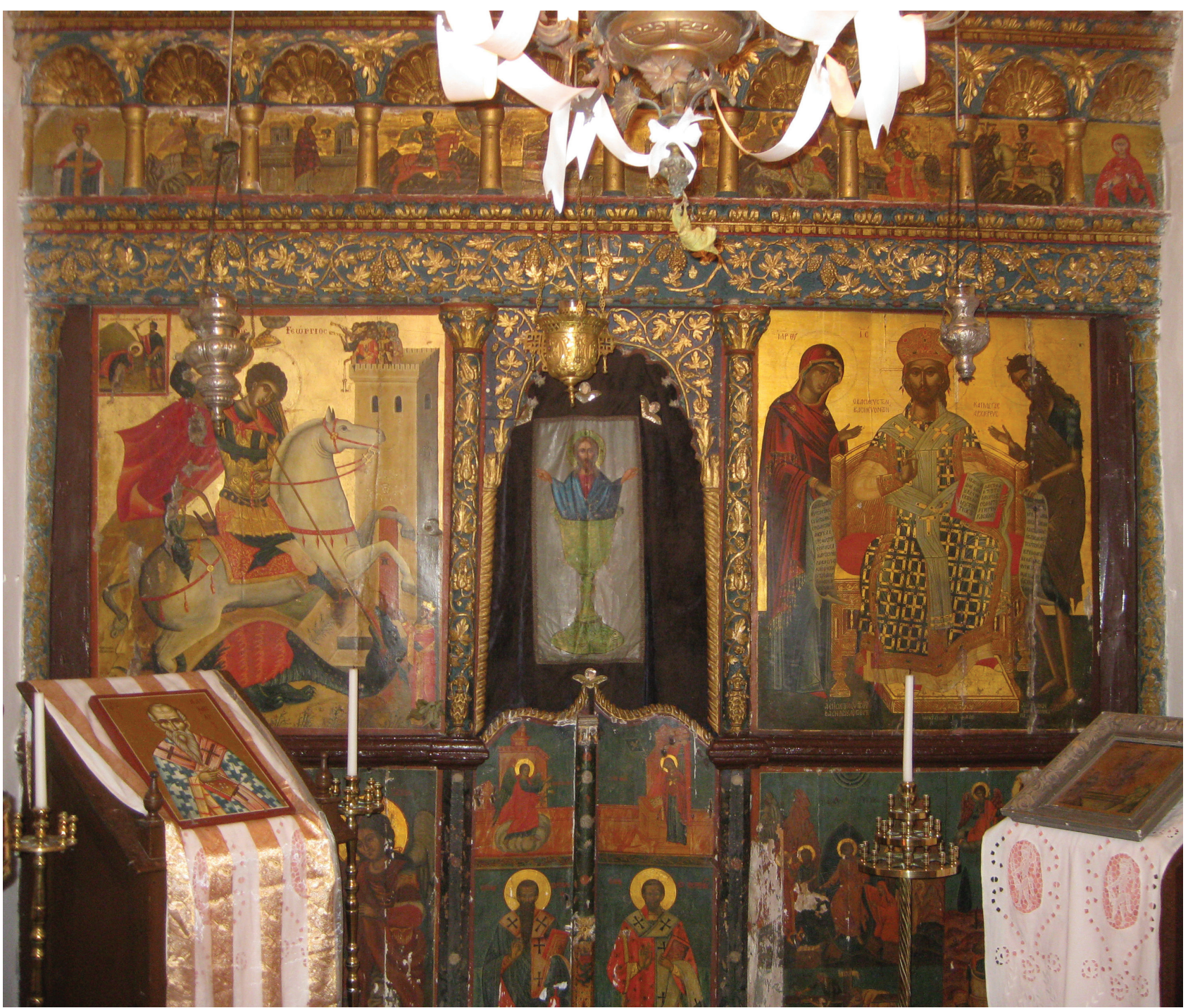

Fig. 15. Siphnos. Templon de Saint-Georges Aphentis 1635.

rouleaux dépliés avec des inscriptions, où le Christ trônant au centre est vêtu du costume de Grand Archiprêtre, tenant l'évangile ouvert avec l'inscription appropriée, comme sur une icône à Patmos datée vers 1600-1610 (Fig. $13)^{45}$ qui se rapproche par ses dimensions $(47 \times 38 \mathrm{~cm})$

\footnotetext{
${ }^{45}$ M. Chatzidakis, The Icons of Patmos, National Bank of Greece, Athènes $1977 / 2$ 1985, 160-161, no 138, pl. 67, 180. Pour le texte de l'évangile, voir ibidem p. 160. On peut déceler les origines byzantines de l'iconographie de la Déis is où le Christ est vêtu en Grand Archiprêtre (Roi des Rois) sur quelques fresques des églises de Kastoria, comme Saint-Athanase Mouzaki (1384/85), l'église de
}

à l'icône de Strasbourg $(48,5 \times 37,5 \mathrm{~cm})$. Nous retrouvons cette iconographie sur une icone à Siphnos $(108 \times 91$ $\mathrm{cm})^{46}$, datée de 1635 et signée par le peintre Constantin

la nonne Eupraxia (1486) et de Saint-Nicolas Magaleiou (1505),

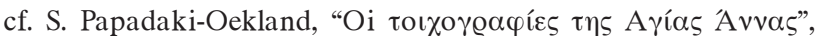
op.cit. (n. 29), 37-38. M. Chatzidakis, S. Pelekanides, Kastoria, Byzantine Art in Greece, Athènes 1985, 106-107, fig. 13 (avec bibliographie).

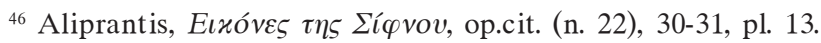
Pour le peintre voir Chatzidakis - Drakopoulou, 'E $E \lambda \eta \eta v \varepsilon \varsigma \xi \omega \gamma \varrho \alpha ́-$ $\varphi o l$, op.cit. (n. 21), 266-267. 
Paleocappas (1634-1640) qui reproduit son modèle avec une grande habileté sur une plus grande échelle (Fig. 14). La Vierge et saint Jean tournés en prière vers le Christ trônant au centre, tiennent des rouleaux dépliés avec le texte de la prière d'intercession traditionnelle.

On ne peut pas éviter la tentation de noter que cette dernière icône, avec celle mentionnée plus haut de saint Georges tuant le dragon, de Zacharias Tzankaropoulos, sont réunies sur le même templon de l'église Saint-

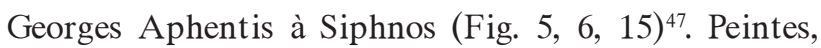
selon les inscriptions dédicatoires, au cours de la même année (1635), elles font partie de la donation d'un même personnage puissant et reconnu de l'île, Vassileios Logothet is ${ }^{48}$, peu après la construction de l'église en 1630 , date gravée sur la plaque de marbre, encastrée sur la façade de l'église, représentant en relief saint Georges tuant le dragon (Fig. 6) ${ }^{49}$.

Sur tous les exemples déjà examinés de la Déisis, où saint Jean tient un rouleau déplié, il se présente vêtu de la mélote et d'un himation court qui laisse ses membres amaigris découverts, évoquant ainsi ses qualités d'ascète du désert. Par contre, sur l'icône de Strasbourg son aspect et ses vêtements correspondent au type iconographique du prophète vêtu à l'antique, attestant ses qualités de prêcheur, repris d'ailleurs sur tous les exemples déjà mentionnés de la Déisis avec le Christ debout (Fig. 9), ainsi que dans la plupart des icônes crétoises des XVe-XVIe siècles, où le Christ est représenté trônant comme sur l'icône des frères Manessis de Venise (1547) (Fig. 10) ${ }^{50}$.

La formule iconographique suivie sur l'icône de Strasbourg se distingue donc des types déjà connus et mérite notre attention, car le peintre s'avance vers une innovation iconographique de la Déisis en réunissant les traits de deux modèles différents, en cours parmi les peintres crétois du XVe au XVIIe siècle. Au type iconographique, où le Christ debout est flanqué de la Vierge et de saint Jean debout et en prière (Fig. 9), mais sans tenir de rouleaux, l'artiste a greffé le motif des rouleaux dépliés avec les textes de la prière d'intercession, rencontré sur une autre série d'icônes de la Déisis où le Christ est représenté trônant au centre de la composition (Fig.

\footnotetext{
${ }^{47}$ Voir plus haut p. 231, notes 21 et 22.

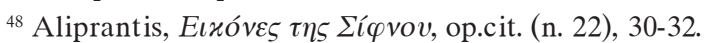

${ }^{49}$ Voir plus haut p. 231-232, note 23.

${ }^{50}$ Voir plus haut p. 238, notes 40 et 41.
}

11-14). Il faut enfin remarquer que le type iconographique de notre icône n'a pas connu de diffusion dans la peinture des icônes des XVe-XVIIe siècles, puisque, jusqu' à présent, nous ne connaissons pas d'exemples reproduisant tous les détails du type iconographique de notre icône.

\section{Quelques remarques sur les inscriptions des rouleaux}

Il est déjà remarqué dans les publications que le texte de la prière d'intercession sur les rouleaux des icônes du musée Byzantin d'Athènes (Fig. 11), du Kunsthistorisches Museum de Vienne (Fig. 12), de Patmos (Fig. 13) et de Siphnos (Fig. 14) reproduit d'une manière générale celui du Manuel de la Peinture de Denys de Fourna ${ }^{51}$ :

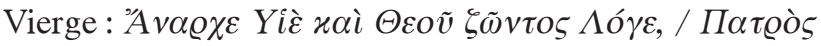

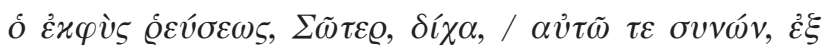

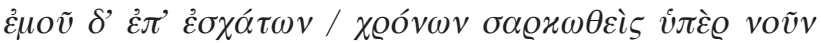

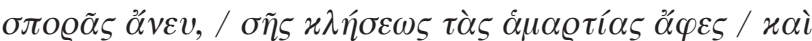

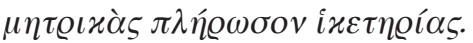

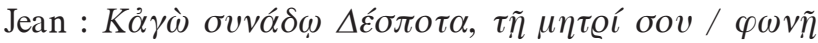

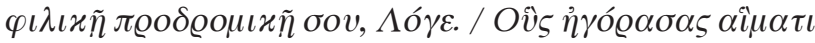

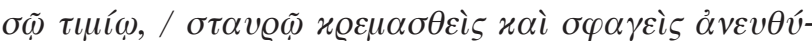

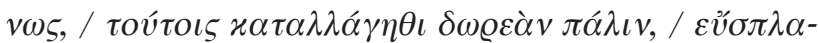

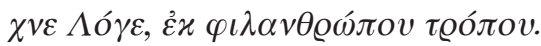

Les textes des rouleaux sur l'icône de Strasbourg (Fig. 7,8$)^{52}$ ne font pas exception à cette règle. Pourtant, une confrontation attentive du texte des inscriptions des icônes sus-mentionnées avec celui de Denys permet de constater quelques différenciations intéressantes.

\section{Icône du musée Byzantin d'Athènes (Fig. 11) ${ }^{53}$ :}

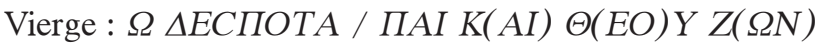

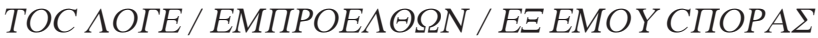

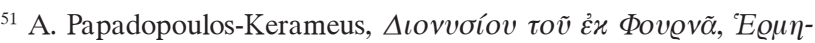

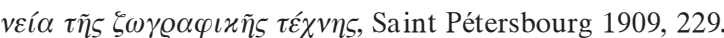

${ }^{52}$ Pour les textes des inscriptions de l'icône voir aussi plus haut p. 235.

${ }^{53}$ Acheimastou-Potamianou, Icons, op.cit. (n. 45), 152-153, no 44.
} 
/ ANEY · EK $\triangle E \Pi(A T) P(O) C$ / ФYC PEYCESC / $C(\Omega T) E P$ IIXA - KAI AY/TS CYNSN OYPA/

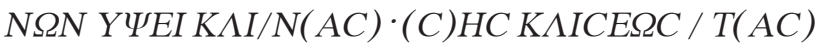

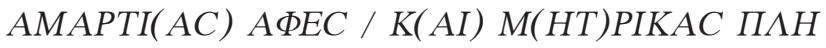
$P \Omega / C O N I K E T H P I / A C$.

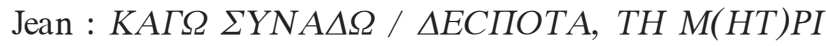

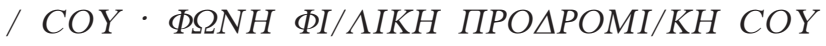

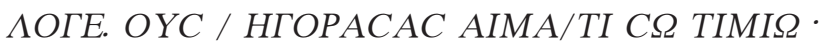

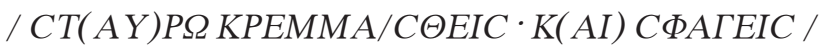

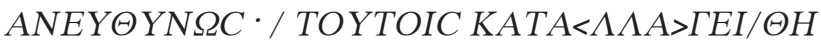

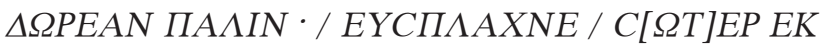

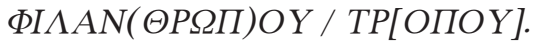

Icône du Kunsthistorisches Museum, Vienne (Fig. 12) ${ }^{54}$ :

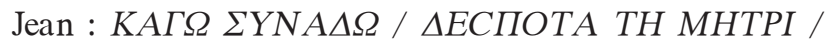

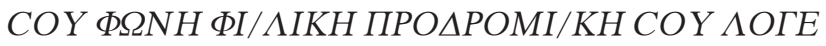

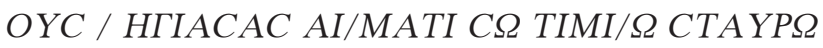

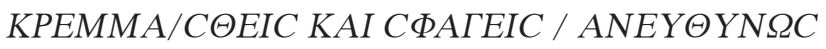

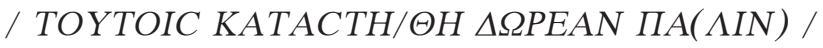
EYCП $\Lambda A X N E C(\Omega T) E P$ / EK [ФI] $\Lambda A N[\Theta P \Omega \Pi] O Y$ ТРОПОY.

Icône de Patmos (Fig.13) ${ }^{55}$ :

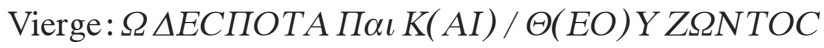

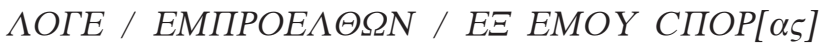
/ ANEY EK $\triangle E$ П(AT)P(O)C / ФYC PEYCESC / $C(\Omega T) E P$ IIXA K(AI) AY/TS CYNSN OYPA/

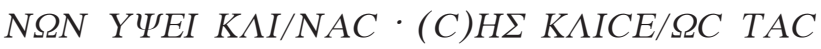
AMAPTIAC / AФEC KAI M(HT)/PI/KAC П H $P \Omega / C O N I K E T H P I / A C$

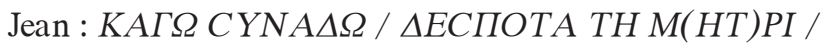

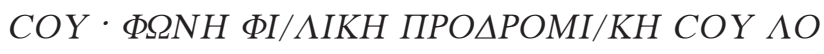
$\Gamma E \cdot O Y C / H \Gamma O P A C<A C>A I / M A T I C \Omega$ TIMI/ $\Omega$

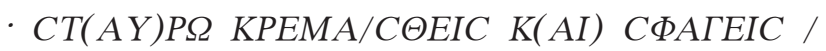

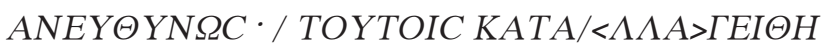

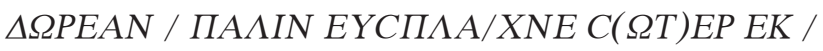

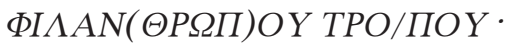

\footnotetext{
${ }^{54}$ Kreidl-Papadopoulos, "Ikonen”, op.cit. (n. 45), 66, 95, no 3, fig. 42.

${ }^{55}$ Chatzidakis, Patmos, op.cit. (n. 48), 163-164, no 138, pl. 67, 180.
}

Icône de Siphnos (Fig. 14) ${ }^{56}$ :

Vierge : $\Omega \triangle$ ЕСПОТА [ПА]I K(AI) / $\Theta(E O) Y \xi \Omega N$

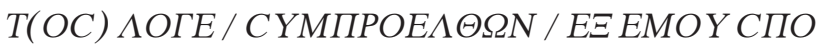
$P(\alpha \varsigma) / A N E Y \cdot E K \triangle E / \Pi(A T) P(O) C \Phi Y C P E Y /$ $C E(\Omega C) C(\Omega T) E P \triangle I X A / K(A I) A Y T \Omega C Y N[\Omega N]$

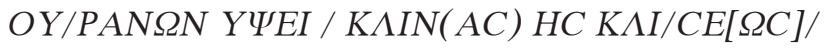
TAC AMAP/TIA(C) AФEC · KAI / M(HT)PIKAC П $А$ TPOCON / IKETHPIAC.

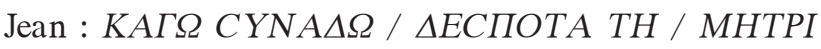

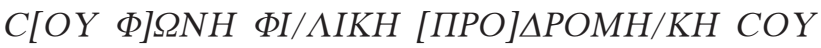

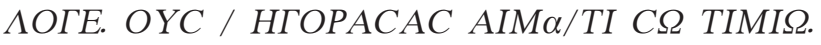

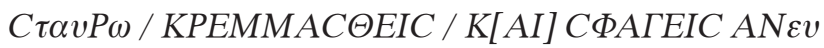

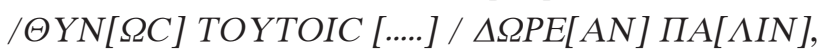
$E Y / \sigma \Pi \Lambda A X N E[C \Omega T] E P$ / K(AI) $\Phi I \Lambda A N(\Theta P \Omega)$ ПОУ ТРОПОУ.

En premier lieu, on doit formuler quelques remarques sur les inscriptions des icônes déjà publiées du musée Byzantin d'Athènes, du Kunsthistorisches Museum de Vienne, de Patmos et de Siphnos.

Prière de la Vierge : (a) Le début de l'inscription de la prière de la Vierge commence par l'invocation " $\tilde{\omega}$

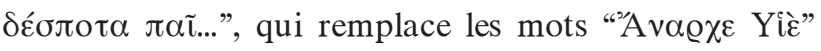
du début du texte de Denys. (b) Le texte des icônes est

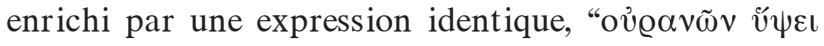
$x \lambda i v a{ }^{\prime}$ ", étendue en trois lignes, qui remplace le texte

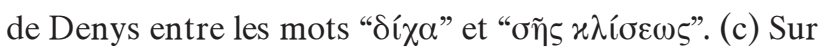

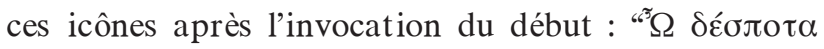

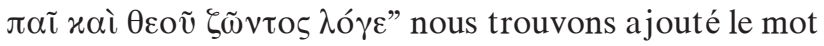

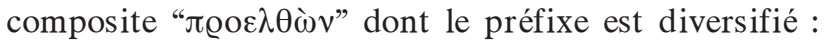

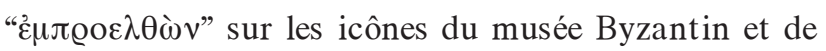

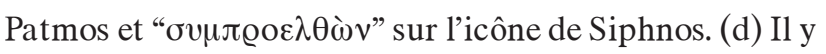

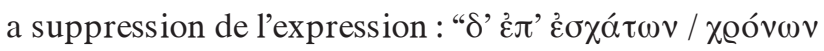

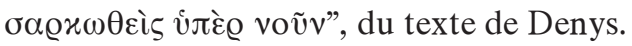

Prière de saint Jean : (a) Le texte suit fidèlement les strophes de la prière de Denys en rajoutant à la fin le mot " $\Sigma \tilde{\omega}[\tau \varepsilon \varrho]$ ", après le mot "Ev̌ $\tau \tau \lambda \alpha \chi \nu \varepsilon$ ", en remplacement du mot " $\lambda o ́ \gamma \varepsilon$ " du texte de Denys. (b) Sur l'icône de

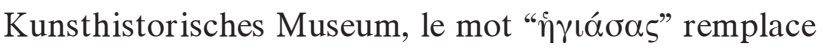

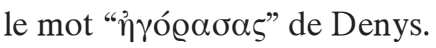

Les textes des inscriptions de l'icône de Strasbourg

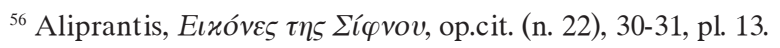


(Fig. 7, 8) suivent de près les versions des icônes mentionnées, mais ils présentent une différence remarquable, puisqu'ils reproduisent seulement les trois premières lignes du texte de Denys, par manque d'espace disponible. En effet les rouleaux ici sont plus étroits et les lettres des inscriptions plus élancées et de plus grandes dimensions de manière que chaque ligne ne peut pas contenir plus de cinq lettres. Par conséquent, alors que les textes des deux prières de l'icône de Strasbourg sont raccourcis, ils s'étendent sur un espace analogue à celui des autres icônes du groupe : treize à quinze lignes pour la Vierge et treize à seize lignes pour saint $\mathrm{Jean}^{57}$. Sur l'icône de Strasbourg le texte du rouleau de la Vierge suit essentiellement la formule des icônes du musée Byzantin, de Patmos et de Siphnos en commençant avec les

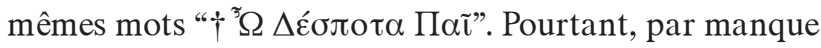

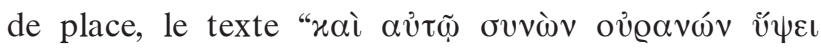
$x \lambda$ íva弓" est ici omis. On peut aussi remarquer que le mot

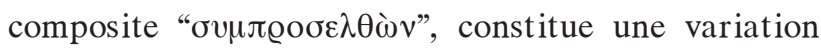

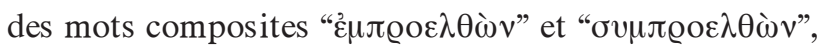
rencontrés respectivement le premier sur les icônes du musée Byzantin et de Patmos et le second sur l'icône de Siphnos. Le texte du rouleau de saint Jean suit fidèlement les trois premières lignes de la prière de Denys, en rajoutant, le mot " $\Sigma \tilde{\omega}[\tau \varepsilon \varrho]$ " au début de la prière, après le mot " $\Delta \varepsilon ́ \sigma \tau o \tau \alpha$ ", à la différence des autres icônes où ce mot est intercalé à la fin de la prière.

Pour conclure, on doit constater que l'ensemble des textes de la prière de l'intercession sur ces icônes offrent une version en partie diversifiée du texte de Denys de Fourna. Nous devons signaler en plus qu'il s'agît de variations, qui montrent une cohérence et un niveau de culture élevé, répondant d'ailleurs à la haute qualité de l'art des icônes étudiées. D'autre part, la répétition des mêmes nouvelles formules d'expression dans ces textes indique la présence d'une rédaction homogène de la prière de l'intercession, diffusée parmi les peintres crétois dès la deuxième moitié du XVe siècle, en conséquence à une époque bien antérieure à celle de la rédaction du Manuel de la Peinture de Denys de Fourna $(1728-1733)^{58}$. Sa diffusion parmi les peintres crétois

\footnotetext{
${ }^{57}$ Les coupures des lignes sont différentes sur chaque icône, adaptées aux dimensions variables des rouleaux et des lettres des inscriptions.

${ }^{58}$ Il s'agit d'une compilation de textes antérieurs réunis pour la
}

fut confortée probablement par son insertion dans un dossier de dessins préparatoires (disegni et anthivola), diffusés en parallèle avec les autres modèles iconographiques du sujet de la Déisis ${ }^{59}$.

\section{Style et conclusions}

Quant à son style, l'icône de Strasbourg d'une exécution technique bien soignée, présente un caractère fidèle à la tradition byzantine tout en adoptant les manières des peintres crétois du milieu du XVIe siècle. Les figures élancées et imposantes du Christ, de la Vierge et de saint Jean sont enveloppées par les larges draperies géométriques, rendues par un dessin incisif et rigoureux. On peut également relever une préférence pour les couleurs limpides et l'emploi du rouge pour le chiton du Christ et le rouge clair pour le chiton de saint Jean ; on perçoit aussi un goût particulier pour les contrastes des tons complémentaires, du bleu foncé et du rouge, pour les vêtements de la Vierge et du Christ. Le traitement souple et la qualité du modelé délicat de la chair, privilégié surtout par les peintres crétois de la deuxième moitié du XVIe siècle, rend aux visages une certaine douceur. Encore, le bord du maphorion de la Vierge, frangé de longs fils d'or et rehaussé par un fin dessin libre de lettres pseudo-coufiques, reproduit d'une manière enrichie, un motif largement diffusé sur les icônes italo-crétoises à partir du XVe siècle ${ }^{60}$. Tous ces éléments traditionnels, infiltrés d'éléments qui trahissent un un goût pour l'art italien, comme l'allure élancée des figures, la douceur des expressions des visages, de même que l'usage abondant des rayures d'or sur l'évangile et le socle du Christ et le fin dessin des fils d'or de la frange de la bordure du maphorion de la Vierge, confèrent un aspect de luxe et permettent de rapprocher l'art de notre icône de celui des peintres crétois du milieu et de la deuxième moitié du XVIe siècle.

première fois par Denys de Fourna, cf. M. Chatzidakis, 'E $E \lambda \eta v \varepsilon \varsigma$

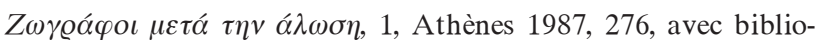
graphie.

${ }^{59}$ N. Chatzidakis, "The Deisis by the Painter Angelos", op.cit. (n. 39), 283-296 (en grec et un résumé en anglais).

${ }^{60}$ Voir exemples cités plus haut note 12 . 
L'icône de la Déisis l'église de Saint-Georges des Grecs, déjà mentionnée à propos de son iconographie qui suit la tradition des œuvres d'Angelos ${ }^{61}$, nous offre en même temps un des meilleurs exemples de l'art des peintres crétois travaillant à Venise vers le milieu du XVIe siècle ${ }^{62}$. Dédiée par les frères Manessis habitants de Venise et datée de 1546 (Fig. 10), cette icône présente plusieurs éléments communs avec la Déisis de Strasbourg. Le Christ trônant au centre de la composition, porte un chiton rouge et un himation bleu foncé comme sur notre icône. Les expressions adoucies des visages, rendus d'un modelé souple rapprochent également l'icône de Venise à celle de Strasbourg. On peut noter aussi des ressemblances pour le rendu du volume des figures élancées de la Vierge et de saint Jean, enveloppées de draperies amples. Nous pouvons également relever l'emploi de combinaisons analogues des couleurs similaires -le rouge et le bleu pour le maphorion de la Vierge et le goût manifeste pour les fins décors en or qui rehaussent les tissus, comme les denses rayures d'or sur l'himation du Christ, ainsi que la bordure du maphorion de la Vierge, décorée d'une frange de fils dorés ; enfin, nous trouvons le même souci pour l'écriture calligraphiée des longues inscriptions sur l'évangile du Christ.

L'icône de Strasbourg peut être rapprochée également d'une autre icône de l'Institut hellénique de Venise représentant le sujet original de la Vierge glorifiée dans le ciel par les prophètes, qui tiennent des rouleaux dépliés en serpentine vers le haut avec des inscriptions calligraphiées $(68 \times 87 \mathrm{~cm})^{63}$. Le donateur qui est figuré sur cette icône, Ioannis Mourmouris, probablement identifié avec un homme de lettres et copiste de manuscrits, installé à Venise vers le milieu du XVIe siècle, est probablement l'auteur des deux inscriptions polystrophes de

\footnotetext{
${ }^{61}$ Voir plus haut p. 238 et note 40.

${ }^{62}$ Chatzidakis, Icônes de Venise, op.cit. (n. 16), 21-22.

${ }^{63}$ Chatzidakis, Icônes de Venise, op.cit. (n. 16), no 10, p. 23-27, pl. III, pl. 8, no 10 , pl. 9.
}

prière originale qui accompagnent cette composition ${ }^{64}$.

Les remarques précédentes montrent que le peintre inconnu de l'icône de Strasbourg, se rattache à la tradition des peintres crétois travaillant à Venise vers le milieu du XVIe siècle. Formé auprès d'un des meilleurs ateliers crétois, il se distingue par son esprit d'innovation dans le choix du type iconographique de la Déisis, et offre l'exemple d'un type rare qui combine les traits de deux formules différentes cristallisées dans la peinture byzantine et chez les peintres crétois vers le milieu du XVe siècle. Le texte des rouleaux ainsi que la calligraphie sophistiquée des lettres des inscriptions montrent en plus un degré d'initiative remarquable par rapport aux autres compositions du sujet connues du XVe siècle. Tous ces traits de l'icône, son iconographie, son style ainsi que ses inscriptions, réunis montrent un peintre de qualité, connaisseur de la tradition et en même temps créatif, se permettant des innovations qui trahissent le haut niveau de culture de son entourage et encore du commanditaire de l'icône ${ }^{65}$.

\footnotetext{
${ }^{64}$ Chatzidakis, Icônes de Venise, op.cit. (n. 16), 26-27.

${ }^{65}$ Pour la clientèle des peintres crétois en Italie voir, M. Chatzidakis, "La peinture de madoneri ou vénétocrétoise et sa destination", Venezia centro di mediazione tra Oriente e Occidente, Aspetti e problemi, Atti del II Convegno Internazionale di Storia della Civiltà Veneziana (Venezia 3-6 ottobre 1973), II, Florence 1977, 675-690.
}

\section{Provenance des figures}

Fig. 1, 7, 8 : Photos Musée de l'Euvre Notre-Dame, Strasbourg. Fig. 2: The Hand of Angelos, op.cit. (n. 14 ) no 37. Fig. 3 : Vocoto-

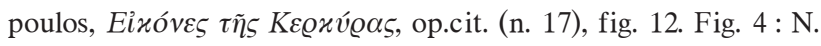
Chatzidakis, From Candia to Venice, op.cit. (n. 12), no 4. Fig. 5, 6, 14, 15 : Photos Nano Chatzidakis. Fig. 9 : Icons of Cretan Art, op.cit. (n. 19), no 116. Fig. 10 : Chatzidakis, Icônes de Venise, op.cit. (n. 16), pl. 6. Fig. 11: Acheimastou-Potamianou, Icons, op.cit. (n. 43), no 44. Fig. 12 : Kreidl-Papadopoulos, "Die Ikonen”, op.cit. (n. 43), fig. 60. Fig. 13: Chatzidakis, Icons of Patmos, op.cit. (n. 45), pl. 67. 


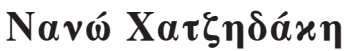

\section{$\triangle$ YO A $\triangle$ HMO $\Sigma$ IEYTE $\Sigma$ KPHTIKE $\Sigma$ EIKONE $\Sigma \Sigma$ TO MOY $\Sigma$ EIO CEUVRE NOTRE-DAME TOY $\Sigma$ TPA $\Sigma$ BOYPГOY: O АГIO $\Sigma$ ГЕ $2 P \Gamma I O \Sigma$ ЕФІППО $\Sigma$ PAKONTOKTONO $\Sigma$ KAI H $\triangle \mathrm{EH} \Sigma \mathrm{H}$}

$\Sigma$

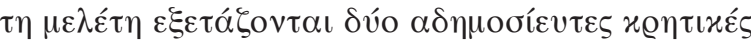

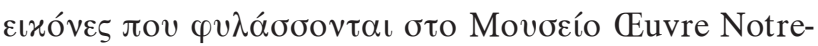

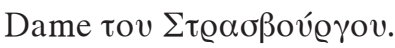

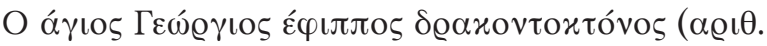

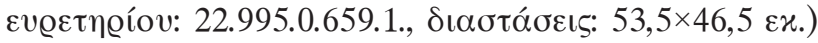

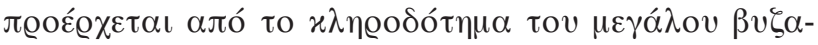

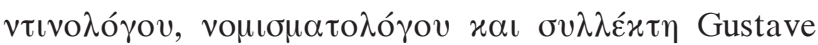

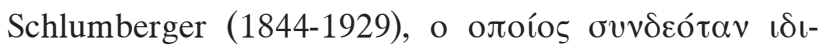

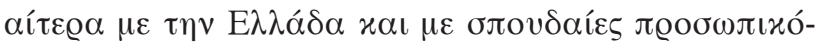

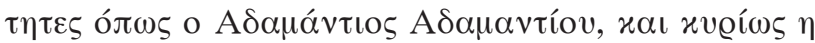

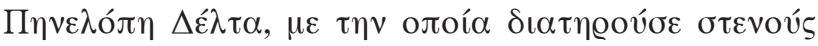

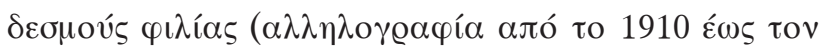

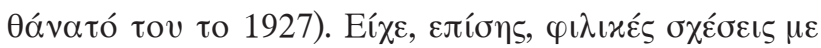

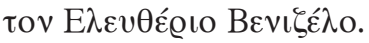

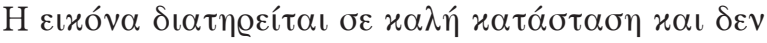

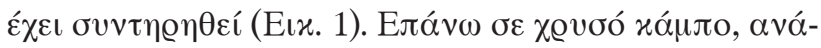

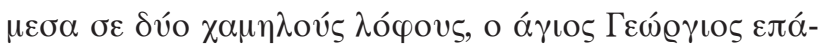

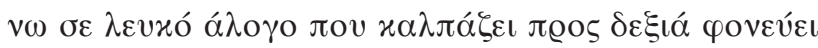

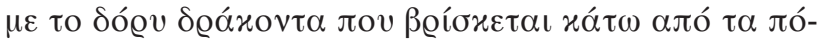

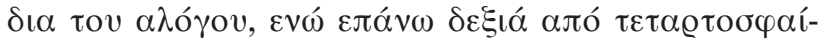

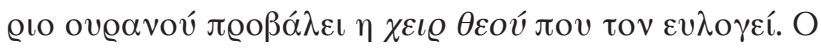

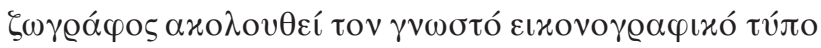

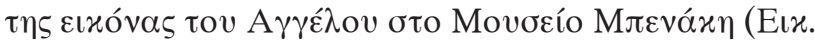

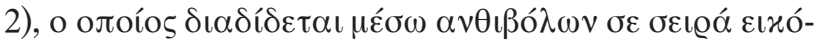

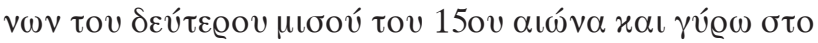

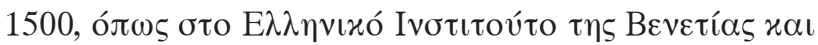

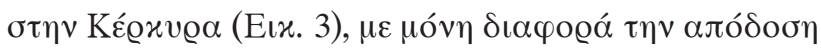

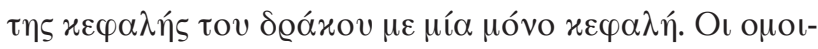

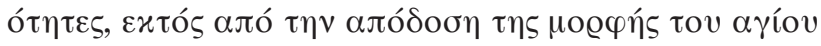

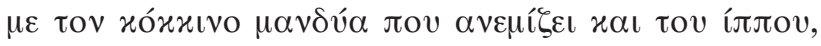

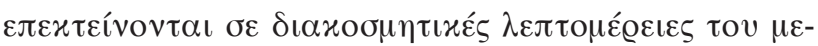

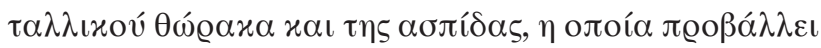

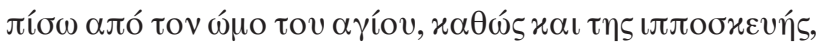

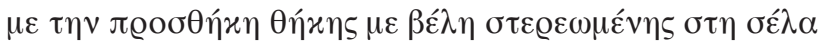

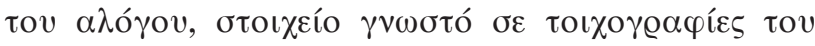

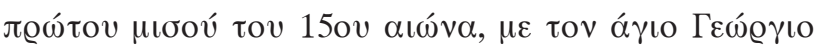

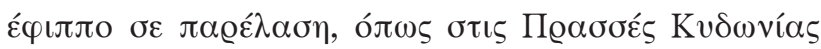

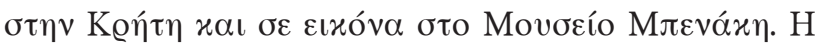

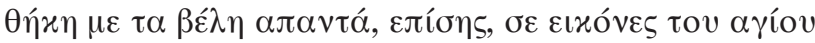

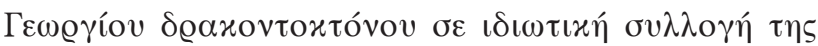

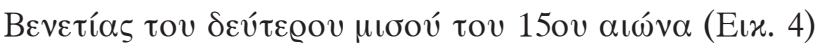

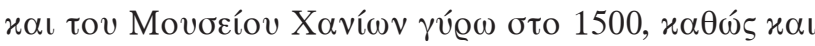

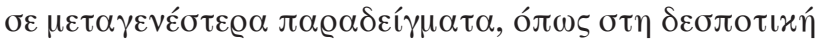

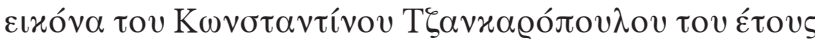

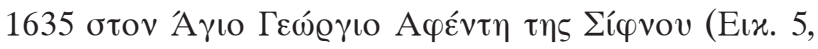

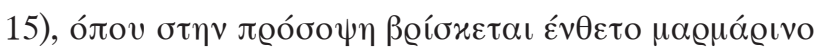

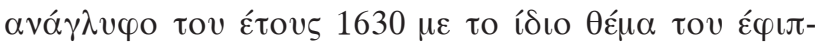

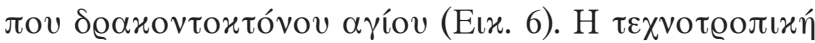

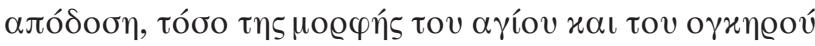

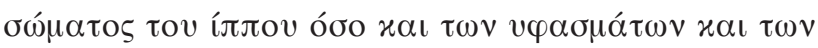

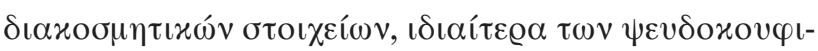

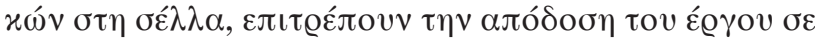

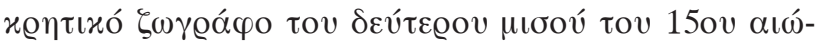

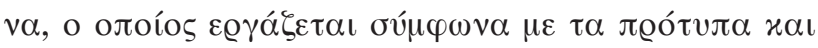

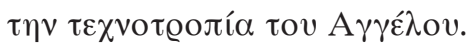

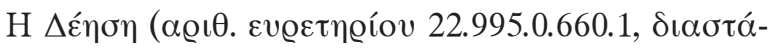

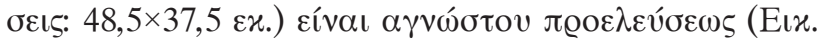

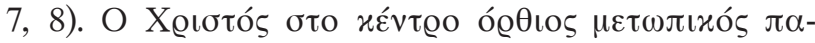

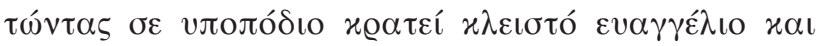

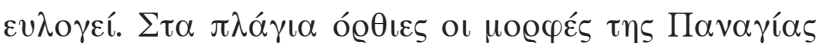

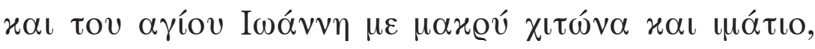

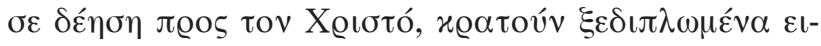

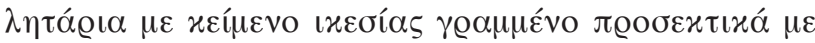

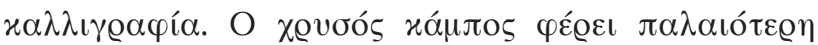

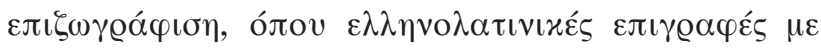

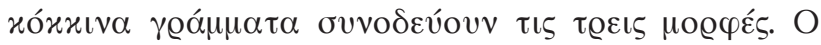

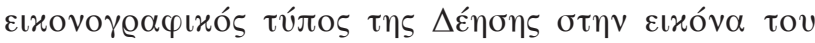

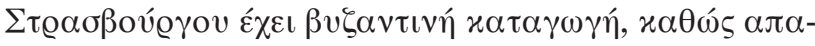

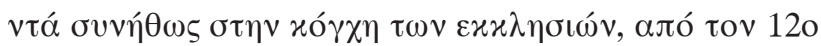

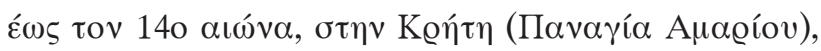

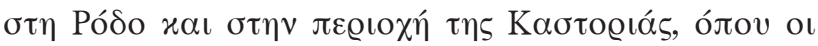


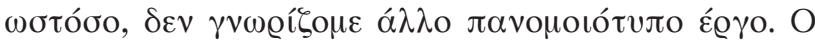

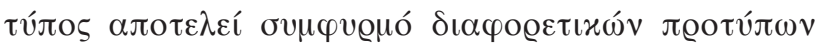

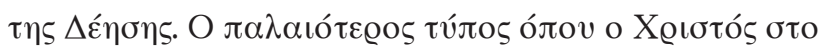

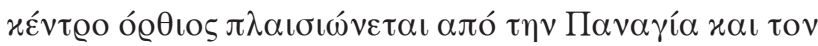




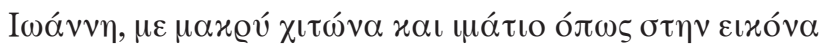

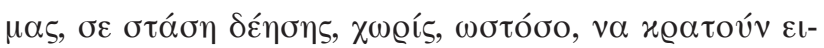

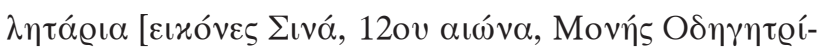

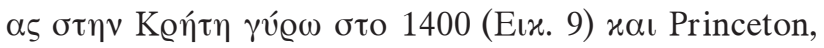

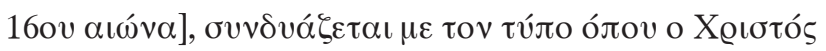

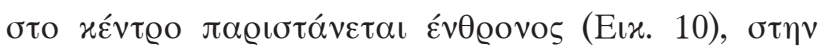

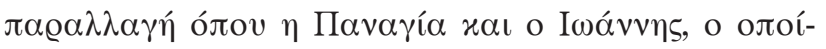

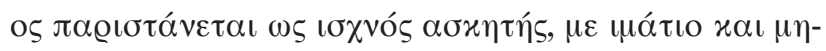

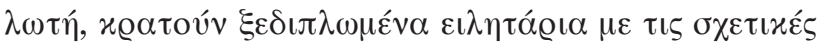

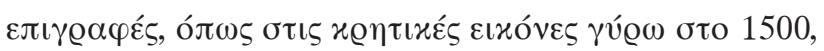

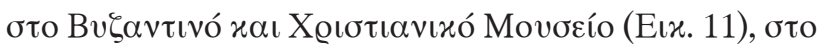

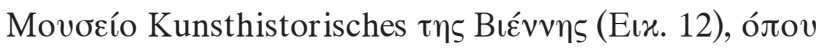

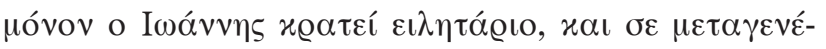

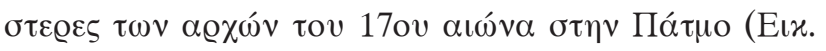

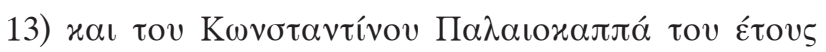

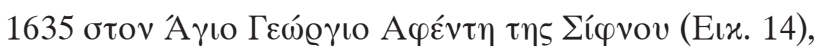

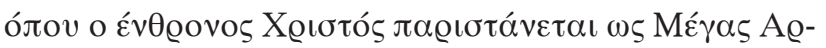

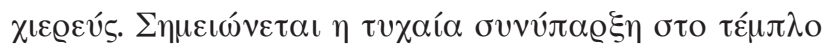

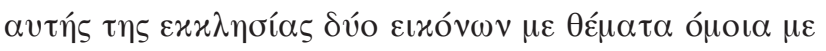

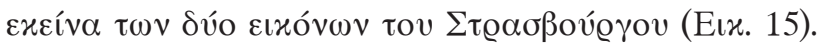

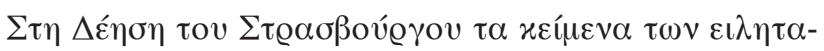

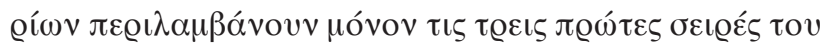

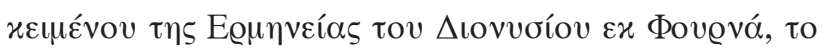

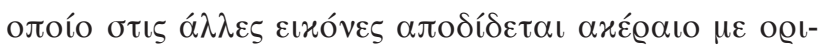

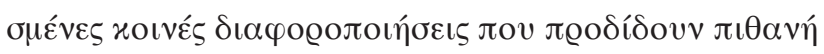

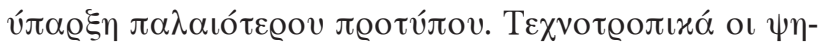

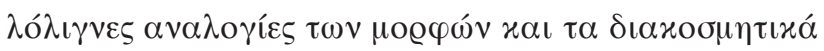

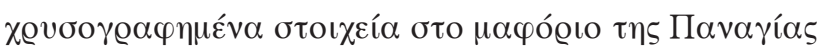

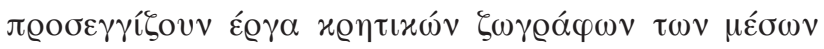

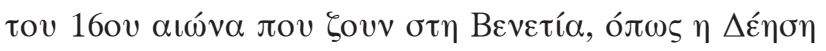

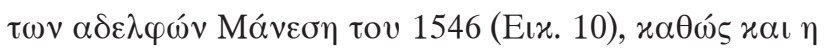

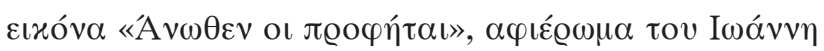

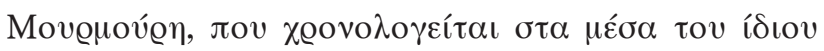

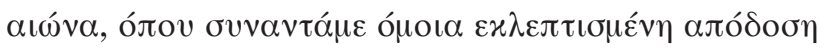

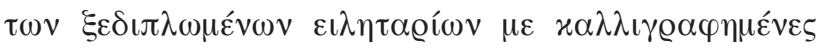

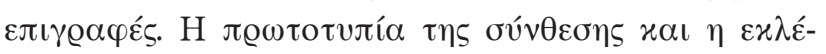

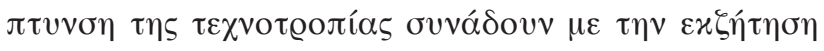

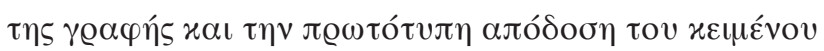

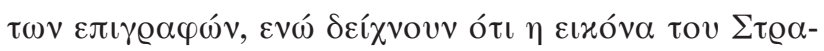

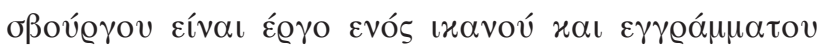

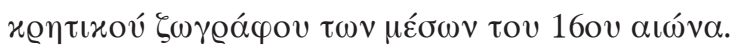

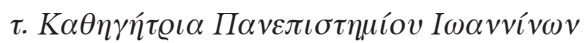
nanochatzidakis@yahoo.gr 